\title{
Using Conflict and Support Counts for Variable and Value Ordering in CSPs
}

\author{
A THESIS \\ SUBMITTED TO THE FACULTY OF GRADUATE STUDIES AND RESEARCH \\ IN PARTIAL FULFILMENT OF THE REQUIREMENTS FOR THE \\ DEGREE OF MASTER OF SCIENCE \\ IN COMPUTER SCIENCE \\ UNIVERSITY OF REGINA
}

By

Ket Wei Yong

Regina, Saskatchewan

February 2016

(C) Ket Wei Yong 2016 


\section{UNIVERSITY OF REGINA}

\section{FACULTY OF GRADUATE STUDIES AND RESEARCH SUPERVISORY AND EXAMINING COMMITTEE}

Ket Wei Yong, candidate for the degree of Master of Science in Computer Science, has presented a thesis titled, Using Conflict and Support Counts for Variable and Value Ordering in CSPs, in an oral examination held on Januiary 21, 2016. The following committee members have found the thesis acceptable in form and content, and that the candidate demonstrated satisfactory knowledge of the subject material.

External Examiner: $\quad$ Dr. Andrei Volodin, Department of Mathematics \& Statistics

Supervisor: $\quad$ Dr. Malek Mouhoub, Department of Computer Science

Committee Member: Dr. Botin Yang, Department of Computer Science

Committee Member: $\quad$ Dr. Samira Sadaoui, Department of Computer Science

Chair of Defense: $\quad$ Dr. Mohamed Ismail, Faculty of Engineering \& Applied Science 


\begin{abstract}
A Constraint Satisfaction Problem (CSP) is a very powerful framework for representing and solving constraint problems. Many real world computational problems in Artificial Intelligence and other areas of computer science can be formulated as CSPs. Problems such as scheduling and timetabling in operations research, map-coloring problem and Boolean satisfiability are some of the examples that can be represented and solved with a CSP framework. Solving a CSP is about searching for a solution in a huge search space. Very often, much search efforts are wasted on the part of the search space that does not lead to a solution. Therefore many search algorithms and heuristic techniques have been proposed to solve CSPs efficiently by reducing the search space. Variable and Value Ordering is one of them. Many experiments and analyses have been conducted to show that good ordering of variables and values can significantly reduce the size of the search space and thus make the search more efficient. Many heuristics have been proposed for ordering variables or values. One such heuristics works by gathering information during search to guide subsequent decision in selecting variables. The heuristic gathers and records information about failures in the form of constraint weight during constraint propagation. Constraints will be assigned weights based on the information gathered. Each variable in the constraint graph will have a weighted degree which is the sum of the weights of the constraints the variable is involved in. In this thesis I will propose a variant of this heuristic where the weight of a constraint is also based on the conflict and support counts of each variable attached to this constraint. The conflict and support counts information is gathered during constraint propagation. The weight of the constraint is the ratio of conflict to support counts. I will also propose a dynamic value ordering heuristic
\end{abstract}


based on the support and conflict count information. Experiments have been conducted on the proposed heuristics using the renowned benchmarks which include random, quasirandom, pattern and real world instances. The test results show that the proposed variable ordering heuristic perform well in the cases of hard random and quasi-random instances. The test results also show that combining the proposed variable and value ordering heuristics can improve the performance significantly in some difficult problems. 


\section{Acknowledgement}

I would like to salute and express my sincere appreciation to my supervisor, Dr. Mouhoub, for his guidance, understanding and empathy during my time in the University of Regina for my Masters' program. I am both honored and grateful to have him as my supervisor without whom I would not be able to complete my Masters' program.

I would also like to thank the Faculty of Graduate Studies and Research and Department of Computer Science for funding my graduate studies in the form of scholarships and teaching assistantships. Without this financial support I would not be able to complete my Masters' program. 


\section{Table of Contents}

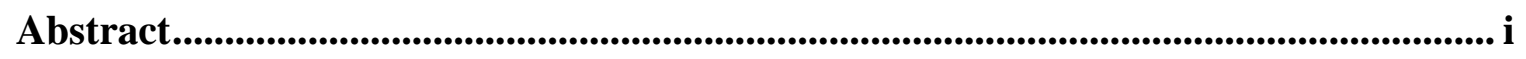

Acknowledgement ....................................................................................................................... iii

List of Tables ...................................................................................................................................... vi

List of Figures...................................................................................................... vii

Nomenclature ................................................................................................ viii

Chapter 1: Introduction ............................................................................................................... 1

1.1 Problem Statement and Motivations ................................................................... 1

1.2 Proposed Solution and Contributions …………………......................................... 4

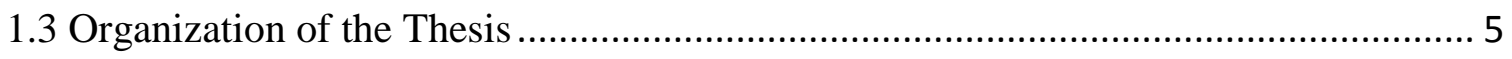

Chapter 2: Background ................................................................................................................ 7

2.1 Constraint Satisfaction Problems ........................................................................... 7

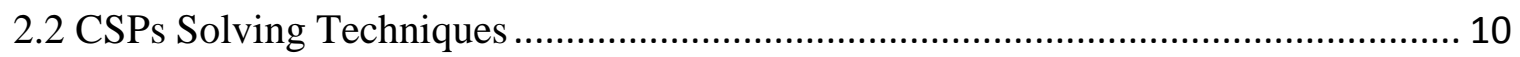

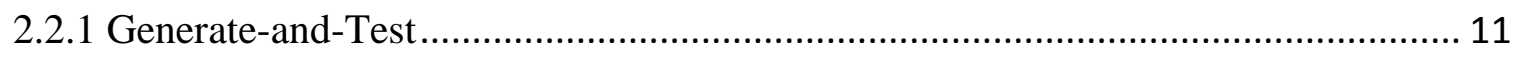

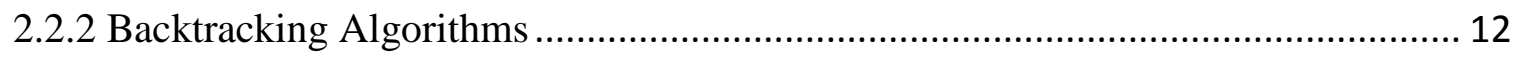

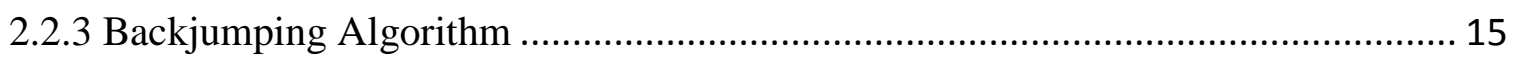

2.2.4 Constraint Propagation....................................................................................... 20

2.2.5 Forward Checking...................................................................................... 22

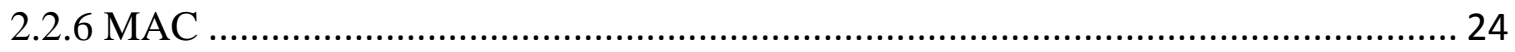

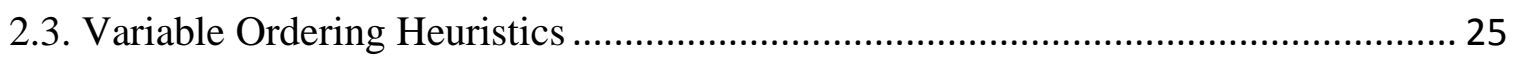

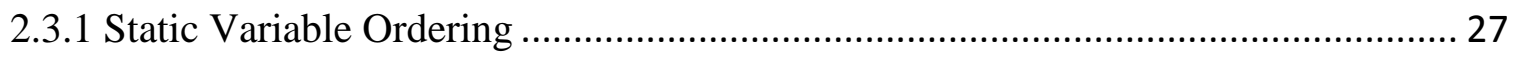

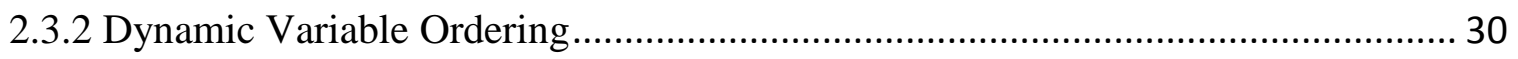

2.4. Value Ordering Heuristics …………………………....................................... 32

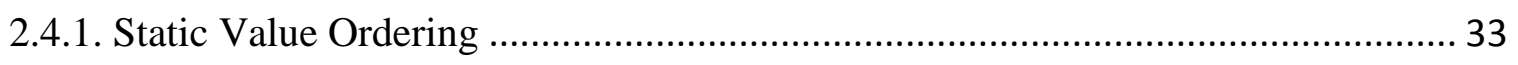

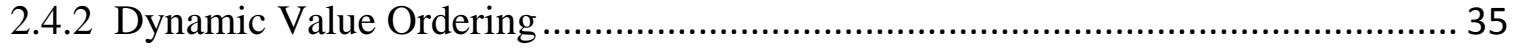

2.5. Ordering Heuristics Based on Information Gathering and Learning ........................ 36

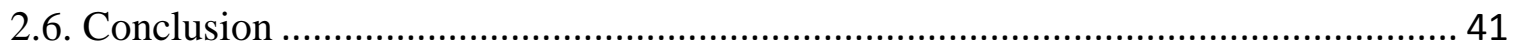

Chapter 3: Proposed Heuristics...................................................................................................... 46 
3.1 The Proposed Variable Ordering Heuristic........................................................ 46

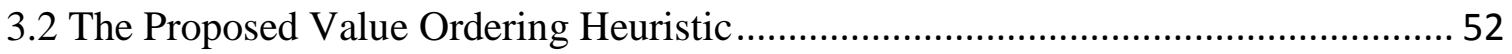

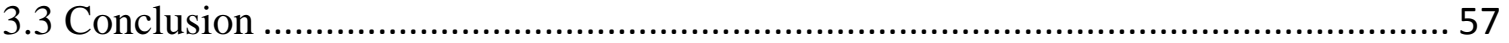

Chapter 4: Experimentation ........................................................................................... 58

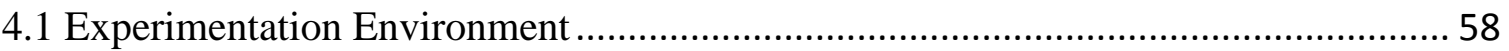

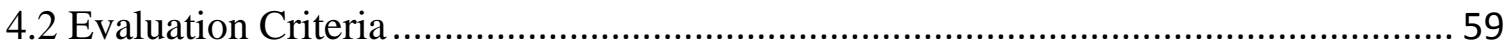

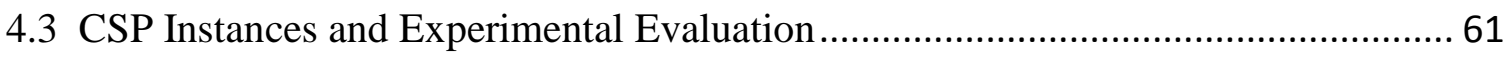

4.3.1 Real World Instances: The Driverlog Problem.................................................... 62

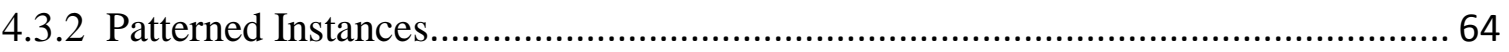

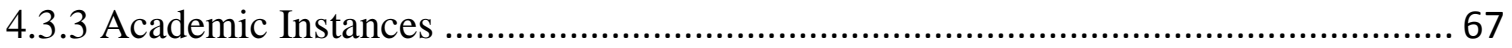

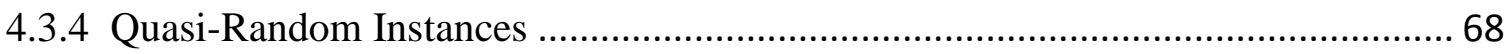

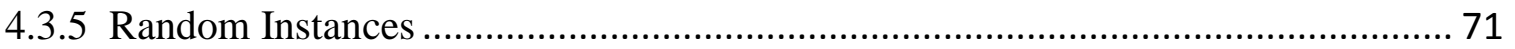

4.4 Performance Evaluation of The Proposed Heuristics .......................................... 75

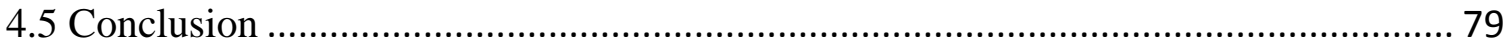

Chapter 5: Conclusions and Future Work ............................................................. 81

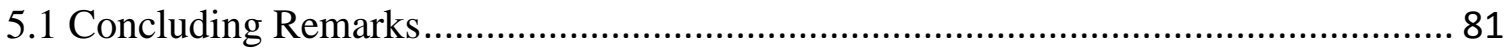

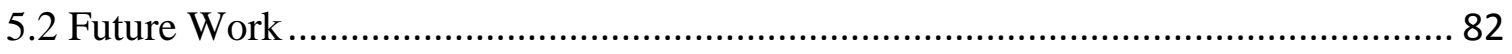

References ...................................................................................................................................... 83 


\section{List of Tables}

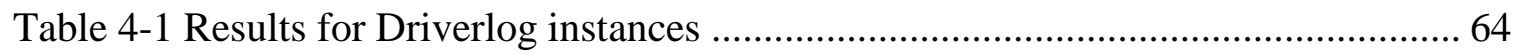

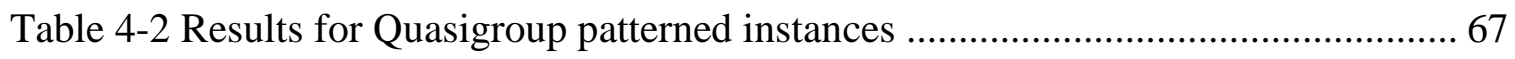

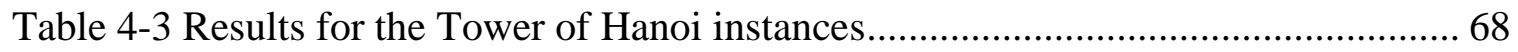

Table 4-4 Results for Quasi-Random instances ........................................................... 71

Table 4-5 Results for forced satisfiable Random instances ....................................... 74

Table 4-6 Results when variable and value ordering heuristics are used together .......... 75 


\section{List of Figures}

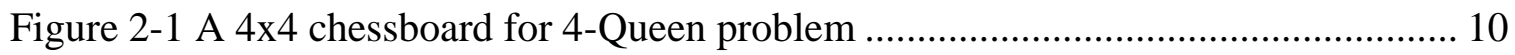

Figure 2-2 A constraint graph representation of the 4-Queen problem .......................... 10

Figure 2-3 Using backtracking algorithm to solve the 4-queen problem (Smith, 1995) .. 13

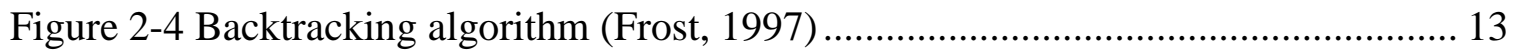

Figure 2-5 A pictorial illustration of thrashing ...................................................... 14

Figure 2-6 A pictorial illustration of the advantage of backjumping............................. 16

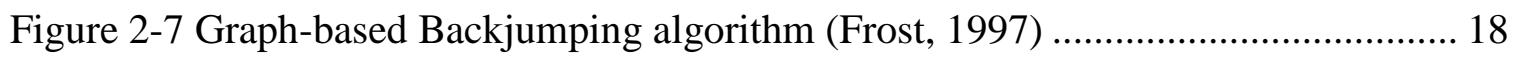

Figure 2-8 A pictorial illustration of conflict set creation .......................................... 19

Figure 2-9 Conflict-directed Backjumping algorithm (Frost, 1997) ............................. 20

Figure 2-10 Solving the 4-Queen problem with forward checking (Bartak, 1998)......... 24

Figure 2-11 Solving the 4-Queen problem using MAC (Bartak, 1998) ......................... 25

Figure 2-12 An instance of the map coloring problem (Jafari, 2010) ............................ 26

Figure 2-13 The search trees for two variable orderings: (B,C,A) and (A,B,C). (Jafari,

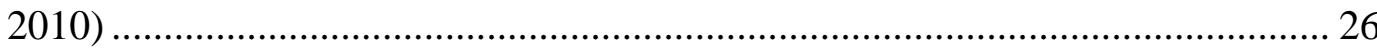

Figure 2-14 Using measurement of propagation power for SVO (Muller, 2005) ............ 29

Figure 2-15 ACO construction graph (Mouhoub \& Jafari, 2011) .................................. 41

Figure 3-1 Pseudo code of the modified revise method .............................................. 50

Figure 3-2 Pseudo code of the modified MAC AC3 method ....................................... 51

Figure 3-3 Pseudo code of the modified forward checking method .............................. 56 


\section{Nomenclature}

$\begin{array}{ll}\text { AC } & \text { Arc Consistency } \\ \text { ACO } & \text { Ant Colony Optimization } \\ \text { CBJ } & \text { Conflict Directed Backjumping } \\ \text { CSP } & \text { Constraint Satisfaction Problem } \\ \text { DVO } & \text { Dynamic Variable Ordering } \\ \text { FC } & \text { Forward Checking } \\ \text { LVO } & \text { look ahead Value Ordering } \\ \text { MAC } & \text { Maintaining Arc Consistency } \\ \text { MC } & \text { Min Conflict } \\ \text { RNDI } & \text { RANdom Information gathering } \\ \text { SVO } & \text { Static Variable Ordering } \\ \text { SLS } & \text { Stochastic Local Search } \\ \text { WNDI } & \text { Weighted Information gathering }\end{array}$




\section{Chapter 1}

\section{Introduction}

\subsection{Problem Statement and Motivations}

A vast number of real world applications in artificial intelligence and computer science can be viewed or formulated as constraint satisfaction problems (CSPs). Constraint satisfaction problem as a powerful framework to solve constraint problem stretches across many domains of study. The countless applications include the field of computer graphic, natural language processing, job shop scheduling, optimization problems in operations research, DNA sequencing in molecular biology, options market in business, symbolic logic, boolean satisfiability, circuit design in electrical engineering ... etc. Because of that, constraint study became a research area that involves researchers from different fields. The research of constraint satisfaction problem in AI began in the seventies and the first constraint logic programming languages were proposed and implemented in the eighties.

A model of constraint satisfaction problem consists of: a set of variables, a domain of values for which the variables can take on and a set of constraints. A constraint is just a logical relation among several variables that restricts the possible values that variables can take. In this paper we concern only about binary constraint, the constraint that involves only two variables, and finite domain. A solution to a constraint satisfaction 
problem is, in its mathematical form, involves finding values assignment for the set of variables in the problem such that all constraints are satisfied.

The usual search techniques used to solve Binary CSPs are either systematic or nonsystematic. Systematic search techniques are complete. They will either find a solution or prove that the problem is inconsistent. However, the drawback of systematic technique is that it may not be practical for large and hard problem. Non-systematic search techniques are incomplete because they may not be able to determine whether the problem is consistent or not. However, non-systematic technique has the advantage of not limited by the size of the problem. Tree search algorithms are systematic search techniques based on depth first search (DFS) or standard backtracking, which are commonly used for solving non-domain specific constraint satisfaction problems with finite domains. Constraint satisfaction problems are often combinatorial in nature. Depth first search or standard backtracking repeatedly selects and assigns a value to a non-instantiated variable until the assignment is consistent or the domain is exhausted. In the case of the domain values exhaustion, it backtracks. Therefore, the standard backtracking search could involve a very huge search space. The worst case time complexity for finding a solution in CSP is of $O\left(d^{n}\right)$. With $d$ being the domain size and $n$ is the number of variables. To improve the efficiency of the search, many search algorithms or heuristics have been proposed. The most commonly seen ones are intelligent backtracking, constraint propagation techniques, variable and value ordering heuristics. Some examples of intelligent backtracking are backjumping, backmarking and dynamic backtracking. Constraint propagation is another commonly seen technique used to improve the efficiency of standard backtracking. Many constraint propagation techniques proposed are dealing with 
search space reduction. These algorithms achieve local consistency by removing inconsistent domain values and thereby reducing the search space. CSP problems are rarely solved by using one heuristic or technique alone. A combination of different techniques is usually applied for solving the problems.

Variable and value ordering heuristics are also very commonly used to improve the efficiency of the tree search. It is believed that good ordering of variables and values may reduce the size of the search space significantly and result in more efficient search. During the search for a solution in CSP using backtracking, a variable is selected at each step and assigned a value from its domain. Selecting which variable to assign a value first can greatly affect the efficiency of the search. The most commonly used heuristic is to select next variable that is most likely to fail first. This is according to the famous failfirst principle (Haralick \& Elliott, 1980). The rationale behind this principle is that if the current partial solution is going to fail, it is better to let it fail early. The sooner it fails the quicker the search can get on the right path to solution. In the case of an inconsistent problem, the inconsistency is proven sooner. Fail-first principle supposes that by triggering early failure, thrashing could be reduced and so the size of the search space. Value ordering, on the other hand, follows the succeed-first principle. For selecting next value to assign to a variable, the heuristic is to select the value that is most likely to be part of the solution. If every value selected is part of the solution then a solution would be found without backtracking. When searching for solution for a hard problem, good value ordering heuristic can have substantial impact on the efficiency of the search because much time is often wasted on searching the sub trees that has no solution. Value ordering heuristic is less proposed in the research community because in complex problem the 
work done to choose a value that is more likely lead to a solution is too costly. It may be better to choose a default or random one. Good value ordering heuristics are generally highly problem specific and general heuristics can be costly (Smith, 1995). Also, it is general belief that if all solutions are required for the CSP or no solution can be found, the order of value is not important.

\subsection{Proposed Solution and Contributions}

This thesis focuses on the variable and value ordering heuristics for constraint satisfaction problems. One dynamic variable ordering heuristic and one dynamic value ordering heuristic are proposed in this thesis.

The proposed dynamic variable ordering heuristic uses a weighted degree heuristic to determine which variable to select next. In weighted degree heuristic every constraint is given a weight based on the weighing heuristic. The constraint weight is then used to determine the order of the variables. In my proposed dynamic variable ordering heuristic, the constraint weight is based on the ratio of conflict to support counts the constraint has on the variables. The conflict and support counts information is gathered during the constraint propagation phase. The heuristic also takes into account when domain wipeout occurs during constraint propagation. Every time when domain wipeout occurs the ratio of conflicts to supports is counted as the domain size of the variable being wiped out. The constraint is then given a weight of the variable domain size.

The proposed dynamic value ordering heuristic also based on the information of the ratio of conflict to support counts. However, in this heuristic the ratio is used to weigh the domain values of variable. Each value is given a weight based on this ratio and the 
weight is used to rank the values in the domain. This ratio is a measure of how likely the value will be part of the solution if it is used to instantiate the variable. This information is gathered during forward checking phase when the inconsistent values of the future connected variables are being weed out.

An experimental study of the proposed heuristics was conducted on some well known instances taken from Lecoutre's website at http://www.cril.univartois.fr/ lecoutre/benchmarks.html. Many of these benchmark instances have been used in various international CSP competitions and renowned research papers. I have compared my proposed heuristic against the well known dom/ddeg or the ratio of domain size to dynamic degree of variable. This heuristic is used because it is easy to implement and very efficient. The experimental results show that my proposed heuristic performs much better than dom/ddeg for most larger and difficult problems of many domains. This is because my proposed heuristic is able to select variables that are most likely to participate in the deadend early on in the search by measuring the conflict to support ratio. This results in the deadend subtree being pruned early and avoid thrashing. The analysis of the experimental results also show that dom/ddeg can degenerate to dom only and perform very poorly in the case where variables have the same degree but the relations variables involved in are of very different constraint of tightness.

\subsection{Organization of the Thesis}

The remainder of this thesis is organized as follow: Section 2 provides a literature review of constraint satisfaction problems and some of the commonly used strategies for solving

them. A formal definition of CSP will be given and some comprehensive examples of CSP will be illustrated. After the literature review of CSP, Section 3 explores in details 
the proposed heuristics. Section 4 elaborates on the experimentation results. Finally, I will conclude the thesis in Section 5 with some prospects of future work. 


\section{Chapter 2}

\section{Background}

This chapter provides some backgrounds and overviews of the study of constraint satisfaction problems. Some standard or well-known algorithms and related concepts for solving CSPs are reviewed. However, more emphasizes and details will be given for those applied or related to this thesis study. Relevant and important definitions will also be provided. This chapter serves as a literature review as well as introduction to constraint satisfaction problems. However, it is by no means a complete literature review or introduction to CSPs.

\subsection{Constraint Satisfaction Problems}

A model of constraint satisfaction problem consists of: A set of variables, a domain of values for which the variables can take and a set of constraints. A constraint is just a logical relation among several variables that restricts the possible values that variables can take. A constraint can be unary, binary or n-ary. In this thesis we concern only about binary constraint or the constraints that involve only two variables and the variables

domains are finite sets. A solution to a constraint satisfaction problem is, in its mathematical form, involves finding values assignment for the set of variables in the problem such that all constraints are satisfied.

\section{Definition 2.1}

A CSP consists of: 
- $\quad$ A set of variables $X=\left\{x_{1}, \ldots \ldots, x_{n}\right\}$.

- $\quad$ A set of domains $D=\left\{d_{1}, \ldots \ldots, d_{n}\right\}$. For each variable $x_{i}$ there is a domain $d_{i}$ with a finite set of values which $x_{i}$ can only take on.

- A set of constraints $C=\left\{c_{1} \ldots \ldots, c_{n}\right\}$, specifies a subset of variables $X$ and restricting the values that the variables can simultaneously take.

Solving a CSP consists of finding assignment of values to all the variables such that all the constraints are satisfied. A constraint is actually a subset of the Cartesian product of $d_{1} \times d_{2} \times \ldots \times d_{n}$ for variables $X=\left\{x_{1}, \ldots \ldots, x_{n}\right\}$ which are compatible to each other. The set of $D_{1} \times D_{2} \times \ldots \times D_{n}$ is also called the state space of the problem.

A constraint satisfaction problem can be represented by a constraint graph or constraint network. Each variable in the constraint satisfaction problem is represented by a node in the graph. An arc connecting two nodes in the constraint graph represents the constraint the variables involve in. The degree of a variable is the number of constraints involving that variable or the number of arcs connected to that variable. A solution to a CSP is an assignment of values to its variables from their respective domain such that no constraint is violated. A binary CSP has at most two variables involve in a constraint. A CSP that has at least one solution is termed as a consistent CSP. A CSP that has no solution is an inconsistent one. Some CSPs may have more than one solution while others may have no solution. One may want to find all the solutions or just one solution with no particular preference as to which one. For CSPs that have no solution, the algorithm for solving it may be adapted to just finding an optimal solution. In such cases, an objective function may be specified and the search is repeated until an optimized solution is found. 
For the purpose of illustration on how a problem can be represented as CSP, I will use the famous Four-Queen puzzle as an example. The Four-Queen puzzle is a challenge for players to arrange four queens on a $4 \times 4$ chessboard so that no two queens are in the attacking positions. An attacking position is such that any two queens are put in the same row, column or diagonal. The Four-Queen problem is just an example of more general NQueen problem where $\mathrm{N}$ queens are to be arranged on the $\mathrm{NxN}$ chessboard. The 4-Queen problem can be represented as a CSP problem as for having a set of variables $X=$ $\left\{x_{1}, x_{2}, x_{3}, x_{4}\right\}$ where each $x_{i}$ represent each of the 4 queens in the problem. The domain of each queen or variable $x_{i}$ is $D_{i}=\{1,2,3,4\}$ which represents each row on the $4 \mathrm{x} 4$ chessboard. The constraint $C_{i j}$ of the problem is such that for any two variables $x_{i}$ and $x_{j}$ ,$C_{i j}$ belongs to the set such that $x_{i} \neq x_{j}$ and $\left|x_{i}-x_{j}\right| \neq|i-j|$. Therefore, in this 4Queen problem: $X=\left\{x_{1}, x_{2}, x_{3}, x_{4}\right\}, D_{i}=\{1,2,3,4\}$ and the constraint rule that restricts the set of allowed pairs is $C_{i j}=\left\{x_{i} \neq x_{j}\right.$ and $\left.\left|x_{i}-x_{j}\right| \neq|i-j|\right\}$. Figure 2-1 is a pictorial illustration of how the 4-Queen problem can be formulated as CSP mentioned above. Each queen is position at each column of the chessboard. We want to move each queen into each row of the chessboard such that no two queens constitute an attacking position. The 4-Queen problem can also be represented as a constraint graph as seen in Figure 2-2. Given the definition of the constraint above for the 4-Queen problem, the allowed pair of domain values between two constraint variables is as follow:

$$
\begin{aligned}
& C_{12}=\{(1,3),(1,4),(2,4),(3,1),(4,1),(4,2)\} \\
& C_{13}=\{(1,2),(1,4),(2,1),(2,3),(3,2),(3,4),(4,1),(4,3)\} \\
& C_{14}=\{(1,2),(1,3),(2,1),(2,3),(2,4),(3,1),(3,2),(3,4),(4,2),(4,3)\} \\
& C_{23}=\{(1,3),(1,4),(2,4),(3,1),(4,1),(4,2)\} \\
& C_{24}=\{(1,2),(1,4),(2,1),(2,3),(3,2),(3,4),(4,1),(4,3)\} \\
& C_{34}=\{(1,3),(1,4),(2,4),(3,1),(4,1),(4,2)\}
\end{aligned}
$$




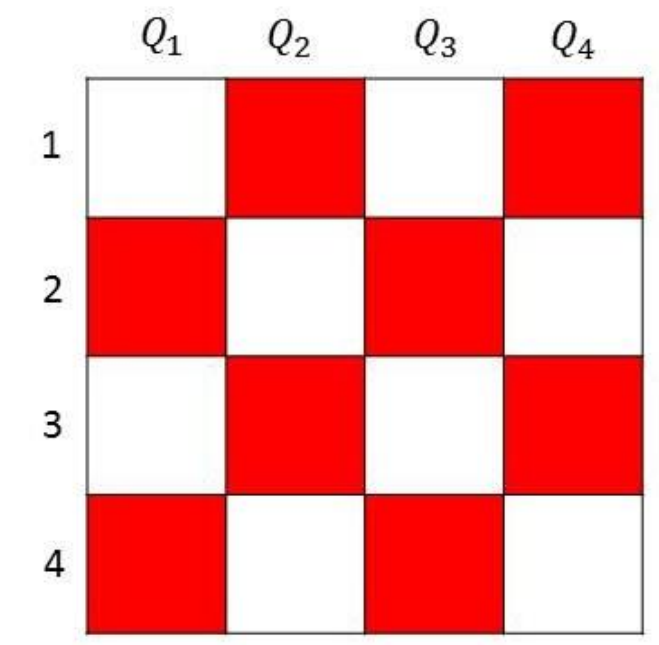

Figure 2-1 A 4x4 chessboard for 4-Queen problem

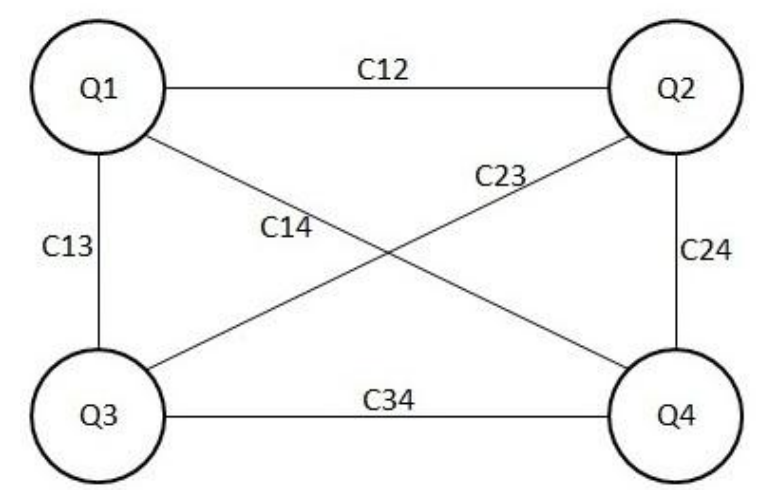

Figure 2-2 A constraint graph representation of the 4-Queen problem

\subsection{CSPs Solving Techniques}

Over the years there have been many algorithms or heuristics proposed to solve constraint satisfaction problems. In this section I will present a few of these search techniques for solving CSPs. Algorithms for solving CSPs can be classified as either 
complete or incomplete. Systematic algorithms are complete algorithms. They guarantee to find the solution if one exists or prove that the CSP is inconsistent. On the other hand non-systematic algorithms are incomplete. They do not guarantee to find the solution if one exists or show that the CSP is inconsistent. For decision problems complete algorithm is required. Examples of systematic algorithms are generate-and-test, backtracking and backtracking with constraint propagation techniques. Examples of incomplete or non-systematic search algorithms are Stochastic Local Search (SLS) methods and evolutionary techniques.

\subsubsection{Generate-and-Test}

Generate-and-Test is possibly the simplest or least sophisticated of all search algorithms. It works by generating all possible combination of assignments for the variables and test at each step to see if any assignment is a solution. However, this method is very inefficient and could be intractable for problems with large search space. The number of possible assignments considered by this method is the size of the Cartesian product of all the variable domains. If a problem has $\mathrm{n}$ variables and each variable has a domain size $\mathrm{d}$, then the possible assignment to the problem is $d^{n}$ or $d$ to the power of $\mathrm{n}$. If there are $\mathrm{c}$ constraint to be satisfied in this problem then there are $c d^{n}$ test to be carried out. If $\mathrm{n}$ is big, this method will become inefficient or intractable. The advantage of generate-andtest is that it is a complete algorithm and for solving simple problems it is very straight forward and effective. However, for complex problem it becomes intractable. 


\subsubsection{Backtracking Algorithms}

Another very commonly used complete method in solving constraint satisfaction problem is backtracking or chronological backtracking. Backtracking search is essentially a depth first search. Backtracking works by incrementally extends a partial solution of consistent current variables set. At each step a variable is instantiated and tested for its consistency with the values in the current partial solution. If the test is successful, the variable is added to the partial solution set. If the test is unsuccessful, the algorithm backtracks from the current variable $v_{i}$ to the most recently added variable $v_{i-1}$ into the partial solution. If there is no consistent value can be found for variable $v_{i-1}$, the algorithm backtracks again to variable $v_{i-2}$. The algorithm is repeated this way until a solution is found. The algorithm stops when it backtracks to the first variable at the root of the tree and found out there is no more value in the domain which can be used to instantiate again. At this point the algorithm proves that the CSP is inconsistent. Figure 2-3 is a pictorial illustration of how backtracking can be applied to the 4-Queen problem. The standard backtracking always returns to the immediately earlier variable in the order of the partial solution set when a dead-end is encountered. It is in this way that it is called chronological backtracking. Figure 2-4 below presents a self-explained backtracking algorithm taken from (Frost, 1997). 


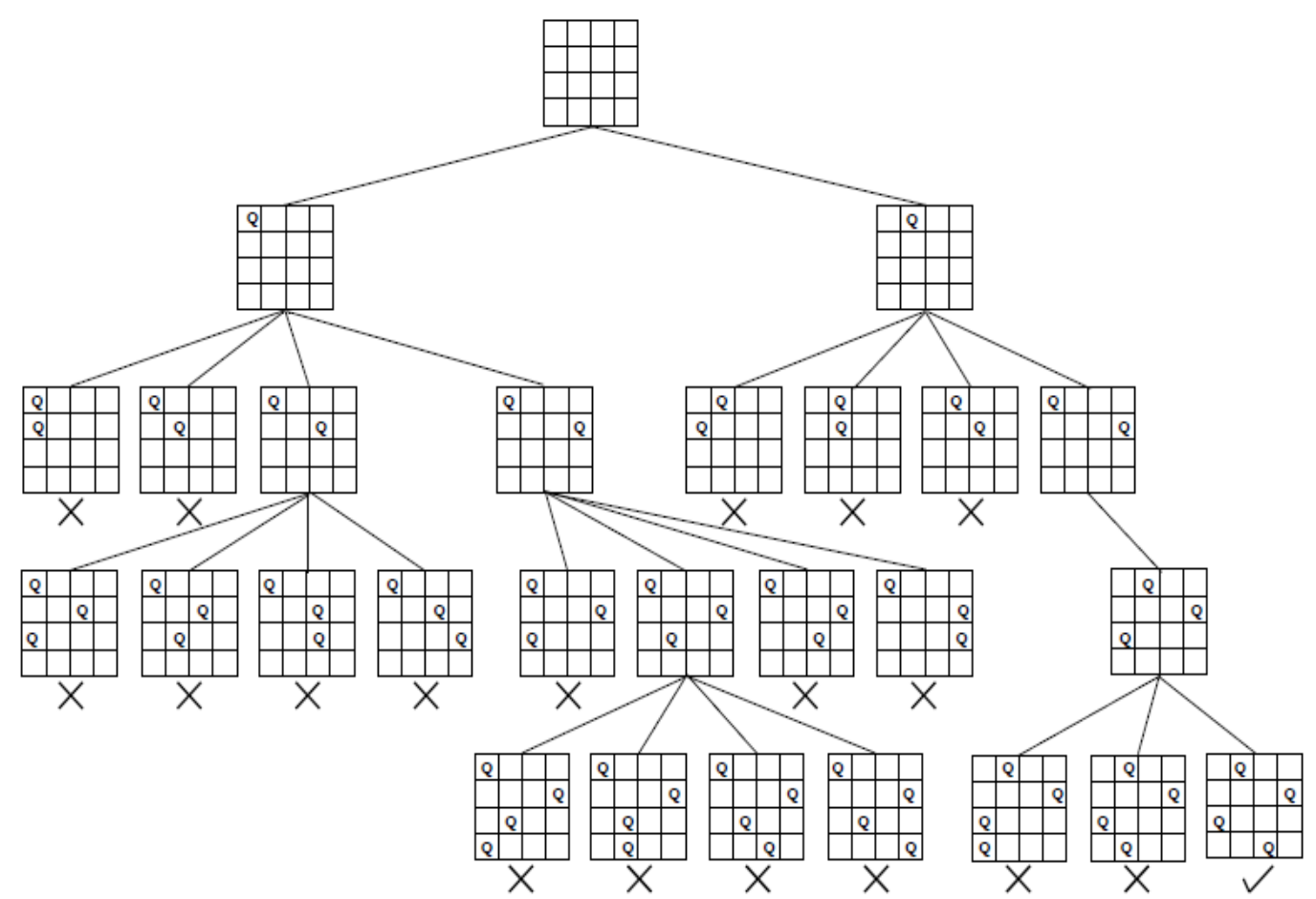

Figure 2-3 Using backtracking algorithm to solve the 4-queen problem (Smith, 1995)

\section{Backtracking}

1. (Step forward.) if $X_{\text {cur }}$ is the last variable, then all variables have value assignments; exit with this solution. Otherwise, set cur equal to the index of the next variable in the ordering. Set $D_{c u r}^{\prime} \leftarrow D_{c u r}$.

2. (Choose a value.) Select a value $x \in D_{\text {cur }}^{\prime}$ that is consistent with all previous variables. Do this as follows:

(a) if $D_{c u r}^{\prime}=\varnothing\left(X_{c u r}\right.$ is a dead-end), go to 3 .

(b) Pop $x$ from $D_{c u r}^{\prime}$ (that is, select an arbitrary value and remove it from $D_{c u r}^{\prime}$ ).

(c) For every constraint defined on $X_{1}$ through $X_{c u r}$, test whether it is violated by $\vec{x}_{c u r-1}$ and $X_{c u r}=x$. If so, go to (a).

(d) Instantiate $X_{\text {cur }} \leftarrow x$, and go to 1 .

3. (Backtrack.) If $X_{c u r}$ is the first variable, exit with "inconsistent." Otherwise, set $c u r$ equal to the index of the previous variable. Go to 2 .

Figure 2-4 Backtracking Algorithm (Frost, 1997) 
The Advantage of backtracking is that it is a complete algorithm and it is at least as efficient as or more efficient than the generate-and-test algorithm. However standard backtracking is still not efficient enough for complex problems. It also has two major drawbacks. One is thrashing and the other is the dead-end is only discovered late in the search. A dead-end node is a node that will not lead to any solution in the search tree. Thrashing is the search keep hitting the dead-end for the same reason in different parts of the search space. This is because standard backtracking only backtracks to the most recently instantiated variable in the search tree which may not be the cause of the conflict. The cause of the dead-end may be many levels up the search tree. For a very huge search space, the same kind of dead-end may be reencountered an exponential number of times before it is finally resolved. Thrashing can be understood easily in Figure 2-5 below.

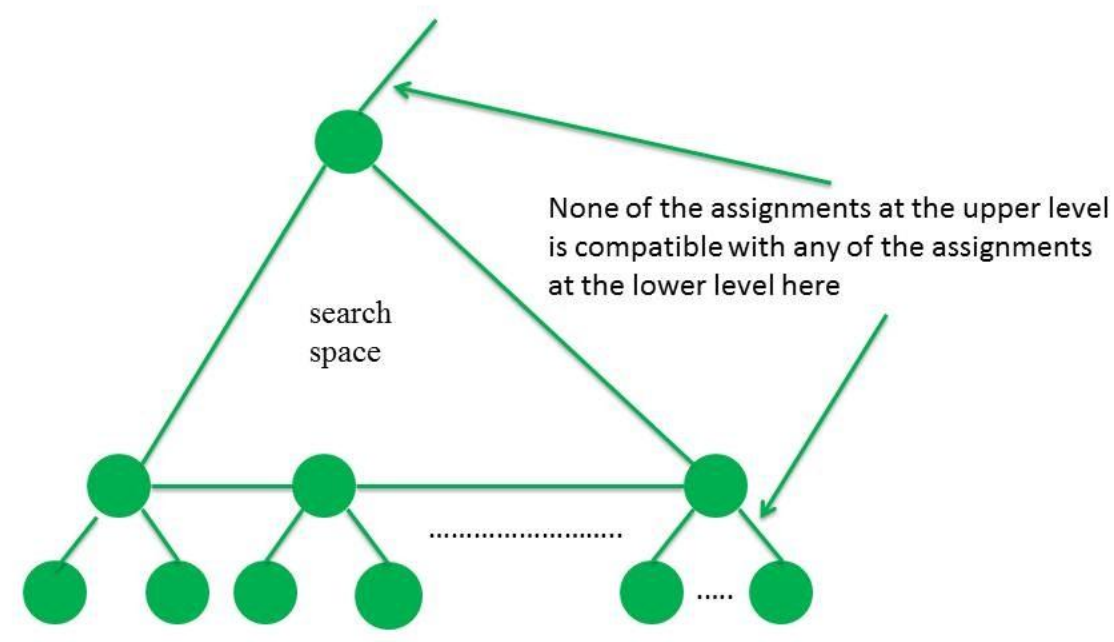

Figure 2-5 A pictorial illustration of thrashing 


\subsubsection{Backjumping Algorithm}

In this section I will discuss another backtracking technique called backjumping. In fact backtracking is central to many algorithms in solving constraint satisfaction problem. There are many variants of backtracking algorithm. These include backjumping, dynamic backtracking, backmarking and variants of backjumping such as conflict-directed backjumping, graph-based backjumping and Gaschnig's backjumping. Generally speaking, these search algorithms can be classified into two broad categories in terms of the scheme they use for checking consistency. They are look-back scheme and lookahead scheme. Look-back scheme is often referred to as backward checking. In this scheme, a variable is instantiated and its value is checked against the past instantiated variables in the search. If the value is inconsistent with the past instantiated variables, another value from the domain is used to instantiate the variable. If no value remains for this variable then a past variable is re-instantiated with a different value. In look-ahead scheme, a variable is instantiated and its value is checked against the future variables for consistency. Standard backtracking, backjumping, dynamic backtracking and backmarking are considered as algorithms that apply the look-back scheme. Forward checking and maintaining arc consistency (MAC) are considered as look-ahead scheme. I will discuss that in the following sections.

Backjumping is an improvement over standard or chronological backtracking because it reduces some of the thrashings from standard backtracking. When a dead-end is encountered in standard backtracking, it always backtracks to the most recently instantiated variable to try to solve the conflict. However, the most recently instantiated variable may not be the one that cause the dead-end. Because of that many useless efforts 
are wasted in trying to solve the dead-end on the wrong variables. In backjumping, whenever a dead-end is encountered, it jumps to the most recent variable that is likely the cause of the dead-end. It is in this way that the thrashing is reduced. Figure 2-6 explains why backjumping is a strategy that can be used to improve standard backtracking. Figure 2-6 is an example of 8-Queen problem. In the figure, after the assignments of $Q_{1}=1$, $Q_{2}=3, Q_{3}=5, Q_{4}=2$ and $Q_{5}=4$, the assignment of $Q_{6}$ has encountered a dead-end. There is no column that $Q_{6}$ can be entered such that it is not in an attacking position. In standard backtracking, the algorithm will backtrack to $Q_{5}$; however, $Q_{5}$ is not the source of the problem. Changing $Q_{5}$ will not resolve the conflict as can be seen in the figure.

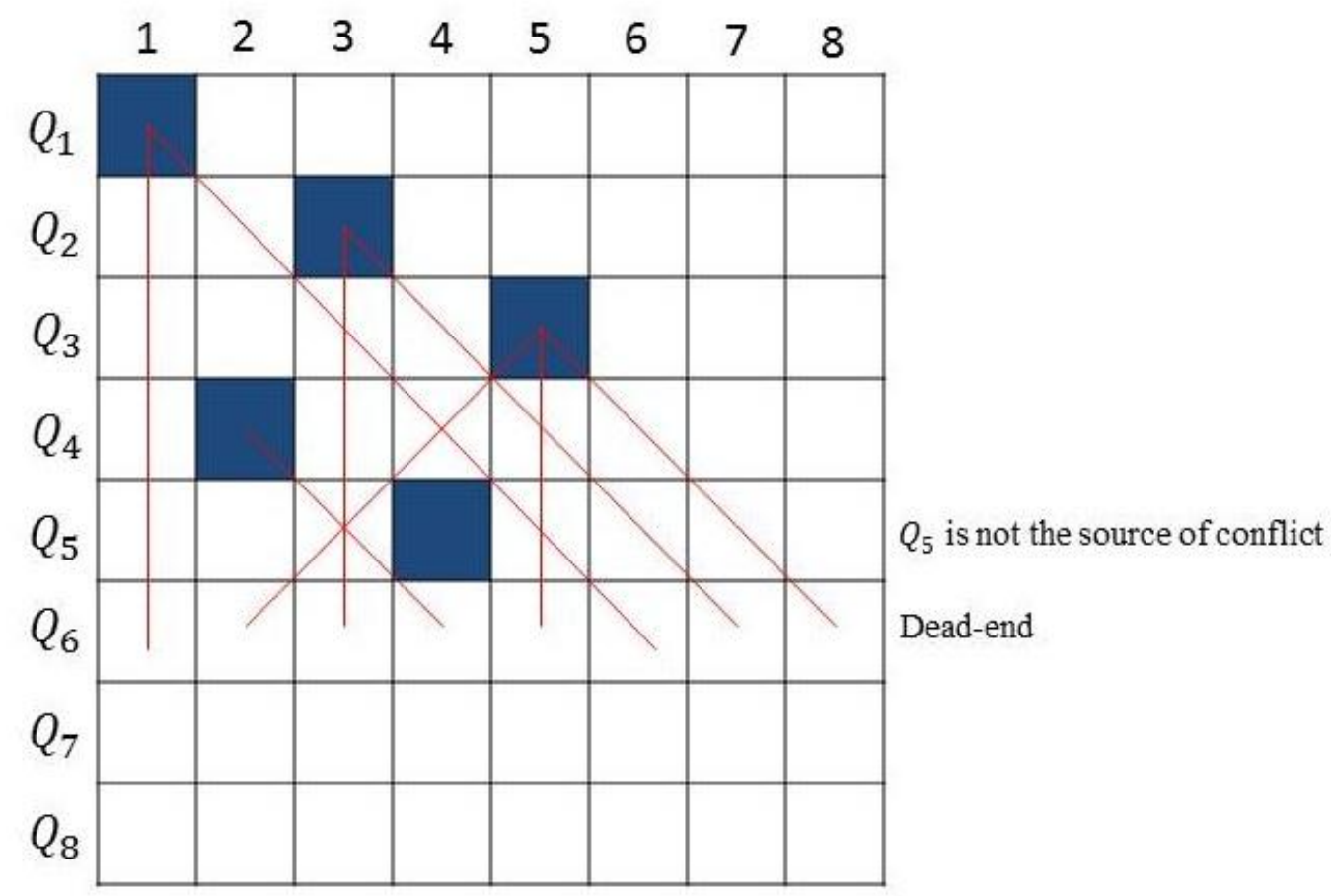

Figure 2-6 A pictorial illustration of the advantage of backjumping

Many research works have been devoted to improve backtracking since it was first proposed many years ago. Many versions of backjumping have been proposed to improve 
backtracking. These include dependency-directed backtracking proposed in (Stallman \& Sussman, 1977), Gaschnig's backjumping algorithm in (Gaschnig, 1978), the graphbased backjumping in (Dechter, 1990) and the conflict-directed backjumping (CBJ) algorithm in (Prosser, 1993). Dependency-directed backtracking of Stallman and Sussman works by discovering and maintaining nogoods during the search and use the information to backjump. Gaschnig's backjumping algorithm is very similar to backtracking except it back jumps from a dead-end node whose children are leaf nodes. Gaschnig's algorithm does not back jump from interior dead-end. For interior dead-end, it chronologically backtracks only.

The graph-based backjumping algorithm by Dechter (Dechter, 1990) works by maintaining a list of nodes used for backjumping. The algorithm can also back jumps from interior nodes. This algorithm maintains a set of parent node for each variable. The parent set of $X_{i}$ consists of all the nodes that share a constraint with $X_{i}$ and precedes $X_{i}$ in the order of instantiation. When a dead-end is encountered at $X_{i}$ and $X_{i}$ is a leaf dead-end, the algorithm back jumps to the latest variable in the parent set. However, if the $X_{i}$ is an interior node, a new set is formed by the union of $X_{i}$ 's parent set and all dead-end variables found below $X_{i}$ in the search tree. The algorithm back jumps to the latest variable in this new set. Figure 2-7 below is the complete algorithm of Dechter's graphbased backjumping taken from (Frost, 1997). 


\section{Graph-based Backjauping}

0. (Initialize parent sets.) Compute $P_{i}$ for each variable. Set $I_{i} \leftarrow P_{i}$ for all $i$.

1. (Step forward.) If $X_{\text {cur }}$ is the last variable, then all variables have value assignments; exit with this solution. Otherwise, set cur equal to the index of the next variable in the ordering. Set $D_{c u r}^{\prime} \leftarrow D_{\text {cur }}$.

2. Select a value $x \in D_{\text {cur }}^{\prime}$ that is consistent with all previous variables. Do this as follows:

(a) If $D_{\text {cur }}^{\prime}=\varnothing$, go to 3 .

(b) Pop $x$ from $D_{c u r}^{\prime}$.

(c) For every constraint involving $X_{c u r}$ and no uninstantiated variable, test whether it is violated by $X_{\text {cur }}=x$. If a constraint is violated, go to (a).

(d) Instantiate $X_{i} \leftarrow x$, and go to 1 .

3. (Backjump.) If $I_{c u r}=\varnothing$ (there is no previous variable in the induced parent set), exit with "inconsistent." Otherwise, set $I_{\text {temp }} \leftarrow I_{\text {cur }}$; set cur equal to the index of the last variable in $I_{c u r}$. Set $I_{c u r} \leftarrow I_{\text {temp }} \cup I_{c u r}-\left\{X_{\text {cur }}\right\}$. Go to 2 .

Figure 2-7 Graph-based Backjumping Algorithm (Frost, 1997)

Prosser's conflict-directed backjumping algorithm (Prosser, 1993) works by maintaining a conflict set used for backjumping. The conflict set maintains a set of variables that are in conflict with some values of the current variable. If the current variable is a dead-end, it jumps back to the nearest variable in the conflict set. Using the 8-Queen problem as an example, Figure 2-8 can serve to illustrate how the conflict set can be created. The conflict set is created by first writing down the variable that is in conflict with the current variable assignment until a consistent value is found. If more than one variable is in conflict with a value, the variable that is furthest away is included in the conflict set. As shown in Figure 2-8, assignment of $Q_{5}=3$ creates a conflict with $Q_{2}, Q_{3}$ and $Q_{4}$. However, only $Q_{2}$ is included in the conflict set. Figure 2-9 taken from (Frost, 1997)is the 
complete algorithm of conflict-directed backjumping. The conflict set for each variable $Q$ is on the right side of the grid in the curly braces.

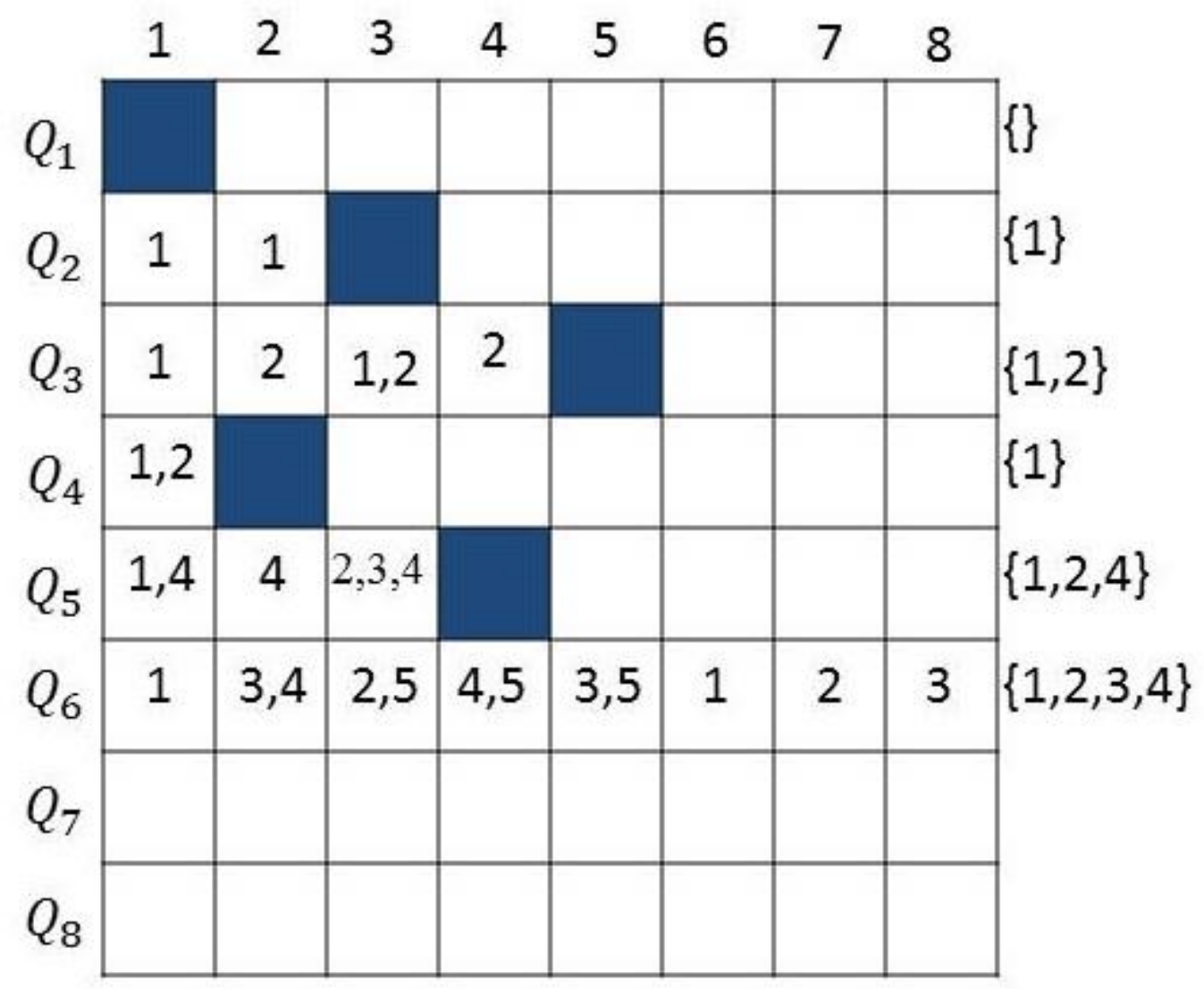

Figure 2-8 A pictorial illustration of conflict set creation 


\section{Conflict-directed Backjumping}

1. (Step forward.) If $X_{\text {cur }}$ is the last variable, then all variables have value assignments; exit with this solution. Otherwise, set cur equal to the index of the next variable in the ordering. Set $D_{\text {cur }}^{\prime} \leftarrow D_{\text {cur }}$. Set $P_{\text {cur }} \leftarrow \emptyset$.

2. Select a value $x \in D_{\text {cur }}^{\prime}$ that is consistent with all previous variables. Do this as follows:

(a) If $D_{c u r}^{\prime}=\varnothing$, go to 3 .

(b) Pop $x$ from $D_{c u r}^{\prime}$.

(c) For $1 \leq \mathrm{i}<$ cur (in ascending order): if $\vec{x}_{i}$ and $X_{\text {cur }}=x$ are inconsistent then add $X_{i}$ to $P_{\text {cur }}$ and go to (a).

(d) Instantiate $X_{\text {cur }} \leftarrow x$ and go to 1 .

3. (Backjump.) If $P_{c u r}=\emptyset$ (there is no previous variable), exit with "inconsistent." Otherwise, set $P \leftarrow P_{\text {cur }}$; set cur equal to the index of the last variable in $P$. Set $P_{\text {cur }} \leftarrow P_{\text {cur }} \cup P-\left\{X_{\text {cur }}\right\}$. Go to 2 .

\section{Figure 2-9 Conflict-directed Backjumping Algorithm (Frost, 1997)}

\subsubsection{Constraint Propagation}

Constraint propagation is a technique besides backtracking used for solving CSPs. Constraint propagation or consistency technique works by making constraint satisfaction problem more tractable by reducing the domain side of the variables. In doing so, some branches of the search tree are pruned and thereby reducing the search space. Many constraint propagation techniques have been proposed by the research community, however very few of them are considered complete. Therefore constraint propagation techniques are seldom used alone for solving CSPs. Constraint propagation techniques are usually applied together with backtracking algorithm to solve constraint satisfaction problems. Like backtracking algorithms, constraint propagation techniques are center to 
the process of solving CSPs. It is very uncommon that constraint propagation technique is not involved in any CSP solving technique.

Constraint propagation techniques are any strategy that removes incompatible values or combinations of values from the domain of variables because of a constraint they involve in cannot be satisfied. Many of the thrashings in standard backtracking can be reduced by applying simple consistency technique. The concept of constraint propagation is easier to understand by using an example. For example, in a game of crossword puzzle, you remove the words India and Japan from the set of Asian countries that can fit in a 5 digit slot because the third letter of the country name must be an ' $i$ '. What you are doing here is called constraint propagation. For a CSP example of two variables $X_{1}$ and $X_{2}$ with the domain values of integer $\{1 \ldots 10\}$, and given the constraint of $X_{1}+X_{2}<5$, the values of $5,6,7,8,9$ and 10 can be removed from the domain of both variables. The reasoning of removing these values from the domain of both variables is an act of constraint propagation.

There are many constraint propagations or consistency techniques proposed by the research community over the last thirty years. In this section I will only explore some of the most commonly used consistency techniques in solving CSPs. Interested reader can find more related materials from (Bartak, 2001) and (Kumar, 1992). The name consistency techniques are born from the concept of a constraint graph used to represent binary CSP as seen in Figure 2 above. The nodes correspond to variables and the edges represent the constraints of the CSP. Take note that arbitrary CSP can be represented as binary CSP. There are two techniques used to convert a non-binary CSP to an equivalent 
binary CSP. They are called dual encoding and hidden variable encoding. I will not explore this advanced topic here. Interested reader can find the material in (Bartak, 2001).

Constraint propagation technique is most commonly applied to enforce node consistency and arc consistency. The requirement for node consistency is that all values in the domain of the variable must satisfy the unary constraint of the CSP. For example, a CSP has a variable $X$ with the domain of $\{1,2,3,4,5\}$ and a constraint of $X<4$. Node consistency will reduce the domain to $\{1,2,3\}$ and the constraint can be dismissed. The requirement for arc consistency for two variables $\mathrm{X}$ and $\mathrm{Y}$ is as follow: For every value $x$ in the domain of $\mathrm{X}$ there exists some value $y$ in the domain of $\mathrm{Y}$ such that $\mathrm{X}=x$ and $\mathrm{Y}=y$ satisfy the binary constraint. However, the concept of arc consistency is directional. The consistency of $\operatorname{arc}(\mathrm{X}, \mathrm{Y})$ does not imply that $\operatorname{arc}(\mathrm{Y}, \mathrm{X})$ is consistent. It must be noted that if a CSP is arc consistent, there is no guarantee that it must have a solution. It is also true that a consistent CSP does not imply arc consistent. Therefore many stronger consistency techniques have been proposed and studied. For this thesis I will not go into the details of it. Readers can find related materials in (Bartak, 2001) and (Kumar, 1992).

\subsubsection{Forward Checking}

In this section I will present a CSP solving technique called forward checking that combine backtracking and constraint propagation. Backtracking and backjumping variants presented above discover conflict by checking the current variable against the previously instantiated variables. Forward checking however, perform checking against the future variables connected to the current variable. Forward checking is good at preventing future conflict. When a variable is instantiated and assigned a value, the 
assignment is used to check against all future variables that are connected to the current variable. Any value from the domain of future variables that is in conflict with the current assignment is removed from the domain. If any of the domains becomes empty as the result of this constraint propagation, it is immediately known that the current search path will not lead to any solution. This branch of the search tree will be pruned immediately. This look-ahead scheme has the advantage of discovering dead-end before reaching the dead-end. It also has the advantage of making sure that the current instantiation is not in conflict with the past variables. Therefore, it is believed that forward checking is always better than the simple backtracking. However, it must be noted that there is an extra overhead for doing constraint propagation for each variable assignment. Figure 2-10 from (Bartak, 1998) below shows the steps taken to solve the 4-Queen problem by using forward checking. Compare this with Figure 2-3 above. It is clear that the number of steps taken is significantly fewer. 


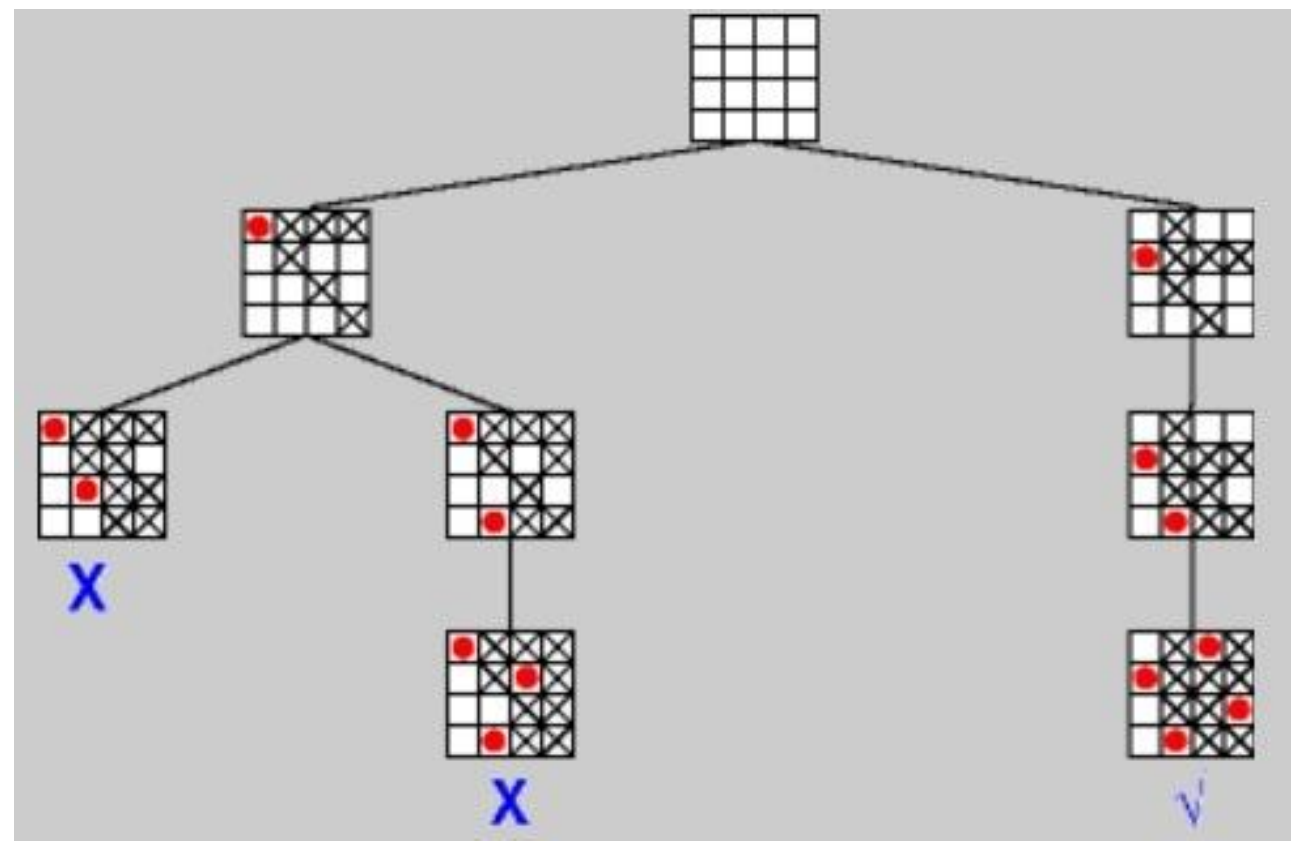

Figure 2-10 Solving the 4-Queen problem with forward checking (Bartak, 1998)

\subsubsection{MAC}

MAC stands for maintaining arc consistency. MAC does even more consistency checks than forward checking. Forward checking enforces consistency between the current variable and future connected variables only. MAC enforces consistency among all future connected variables starts from the current variable. The advantage is that more fruitless branches will be pruned earlier and resulted in even smaller tree. However MAC will incur even more overhead by doing the extra consistency check. Figure 2-11 below from (Bartak, 1998) shows the steps taken to solve the 4-Queen problem using MAC. Compare that with Figure 2-10 above. It is clear that an even smaller tree is resulted and fewer steps are required to reach at the solution. 


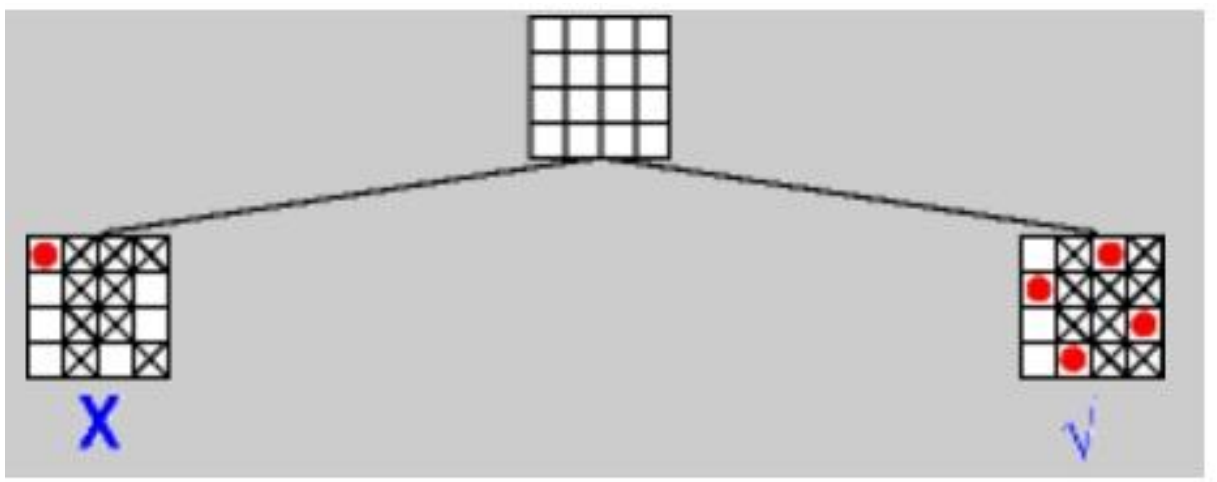

Figure 2-11 Solving the 4-Queen problem using MAC (Bartak, 1998)

\subsection{Variable Ordering Heuristics}

Constraint satisfaction problems are often solved by using systematic backtracking algorithm. In this algorithm a variable is chosen and instantiated by assigning a value to it. If the instantiation succeeded, the algorithm proceeds to select another variable. If the instantiation failed to satisfy the constraint, the algorithm backtracks. If backtracking algorithm is used to solve constraint satisfaction problem, then the next question to ask is which variable should be chosen to instantiate first. Many experiments have been done by researchers with heuristic proposed in this field. These experiments show that variable ordering can have very substantial impact on the size of the search space (Boussemart et 2004; Freuder \& Quinn, 1985; Haralick \& Elliott, 1980).

Good selection of variable and value can lead to the solution quickly. This fact can be easily demonstrated with an example taken from (Jafari, 2010) as illustrated in the Figure 2-12 and 2-13 below. Figure 2-12 shows an instance of a map coloring problem where each node has a domain value of $\{r, b\}$. Figure 2-13 shows two variable ordering $(B, C$, A) and (A, B, C). It is clear that the ordering (A, B, C) ends up with a smaller search tree. 


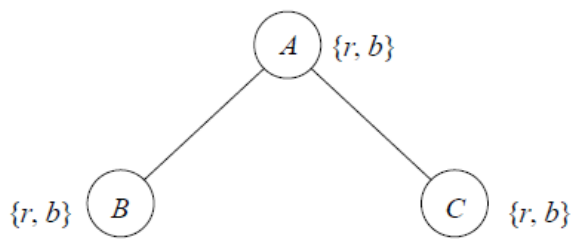

Figure 2-12 An instance of the map coloring problem (Jafari, 2010)
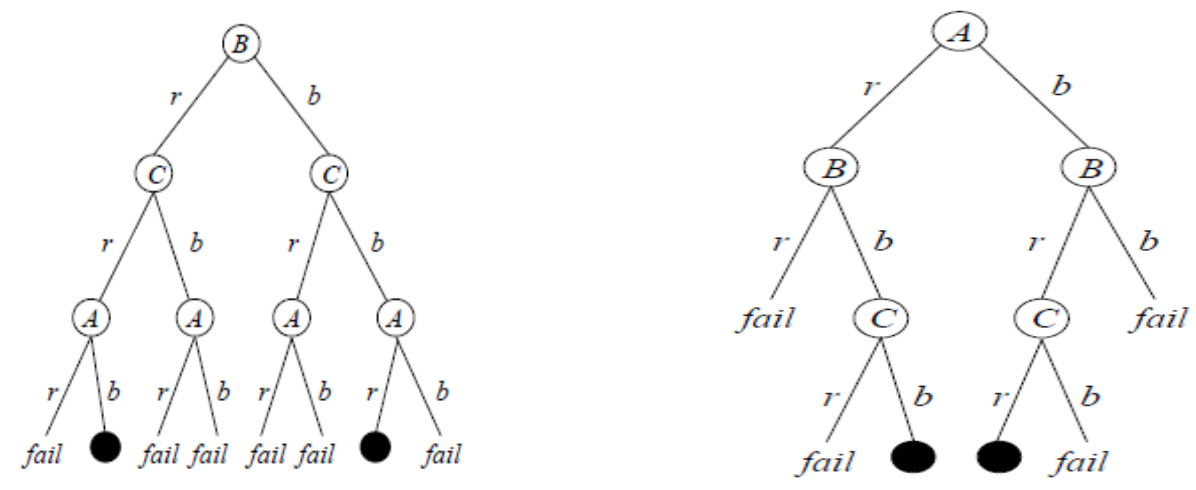

Figure 2-13 The search trees for two variable orderings: $(B, C, A)$ and $(A, B, C)$. (Jafari, 2010)

Many heuristics on variable ordering have been proposed by the research community in the past. Variable ordering heuristic generally fall into two categories: Static variable ordering (SVO) and dynamic variable ordering (DVO). Static variable ordering specifies the order before the search begins and the order does not change after that. Dynamic variable ordering orders the variables during the search based on the current state of the search. The most common heuristics used to select variable order are based on the failfirst principle (Haralick \& Elliott, 1980). This principle selects the variable that is most likely to fail first. The reason behind this is if the current partial solution is going to fail, it is better to let it fail early. The sooner it fails the quicker the search can get on the right path to solution. Fail-first principle also supposes that by triggering early failure, the depth of the search tree can be reduced. 
The reason behind ordering the variables is to obtain a good order of variables so that it will reduce the search space and hopefully make the search more efficient. However, what is considered a good ordering depends very much on the situations. In general, it depends on the structure or character of the problem at hands and the behavior of the applied solution methods. Static variable ordering (SVO) deals with the character or structure of the problem to try to come up with a good ordering. Therefore, a good static variable ordering heuristic is very problem specific. Dynamic variable ordering (DVO) deals with the search behavior. DVO bases on the current states of the search to reorder the variables. Therefore the order of the variables may be different from one branch of the tree to the other. Sometimes SVO and DVO can be used together to solve a problem.

\subsubsection{Static Variable Ordering}

Many static variable ordering (SVO) heuristics have been proposed by the research community in the past. I will only present a few in this section. Most of these heuristics are based on the fail-first principle mentioned above and ordered the variables according to the size of the domain or the amount of constraints the variable involves in. Smallest Domain First is a SVO that orders the variable according to the size of the variable domain. The variables that have the smaller domain size are instantiated first before the variables that have the larger domain. The reasoning behind this is: assuming everything else being equal, variable with the smaller domain size is more likely to fail first. Variables with smaller domain size also have fewer subtrees attached to it. Maximum degree, deg, used in (Frost \& Dechter, 1994) is an ordering heuristic that chooses the variables with maximum degree to instantiate first. The degree of a variable is the number of other variables attached to it. Some heuristics combined both the size of the 
domain and the degree of variable. Dom/deg proposed in (Bessiere \& Regin, 1996) uses the ratio of domain size to the degree of variable to order the variables. The heuristic selects the variable with the smallest ratio to instantiate first. It is not difficult to see that combining the domain size and degree of a variable may be more desired than using only one of them to determine the ordering. Imagine a situation where $x_{i}$ has a domain size of 20 and a degree of 1 only whereas $x_{j}$ has a domain size of 19 and a degree of 20. If SDF is used, $x_{i}$ will be chosen first to instantiate in the ordering. However, this is not a better choice of ordering the variables. Therefore, it may be more desired to combine both of them and use them equally.

As mentioned above, static variable ordering deals with the character or structure of the problem. The problem structure is studied and the variable is ordered before the search begins. Therefore, SVO can use any backtracking algorithm. But what is considered an initial good ordering differs for different people and problem. (Muller, 2005) proposed to propagate as much as possible and as early as possible. This idea favors variables that cause a lot of propagation to go first in the ordering. Variables that cause less propagation will be instantiated later. Therefore a measurement of propagation power is needed to order the variables. He uses variable's dependencies on other variables as a measurement of propagation power. The idea of variable's dependencies here is very flexible. It can be interpreted differently for different problem space and level of abstraction. Based on CSPs represented as a constraint graph, a variable's propagation power can be measured according to the number of constraints it involves in. Using adjacency matrix as a data structure to represent dependencies of the variables, many SVO can be obtained by analyzing the graph. Figure 2-14 from (Muller, 2005) is an example of two SVOs based 
on the measurement of propagation power. Figure2-14(a) shows a graph representation of a CSP with 5 variables. Figure 2-14(b) shows how to measure the propagation power based on a variable's degrees. The SVO ordering is therefore $\left\{v_{2}, v_{3}, v_{0}, v_{1}, v_{4}\right\}$. Figure 2-14(c) with different analysis shows a different ordering of SVO $\left\{v_{4}, v_{0}, v_{1}, v_{3}, v_{2}\right\}$ can be obtained by using the Floyd-Warshall algorithm. Floyd-Warshall calculates the shortest paths between nodes. The algorithm prefers the isolated nodes in the ordering because they have longer paths than variables with more connections. This example illustrates that SVO ordering is very dependent on the structure of the problem and analysis applied to it.

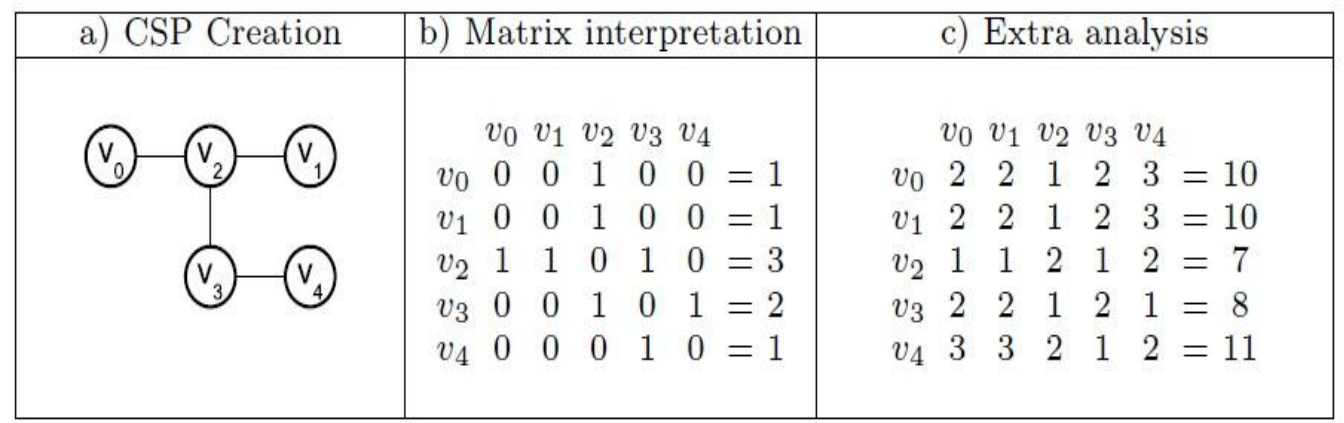

Figure 2-14 Using measurement of propagation power for SVO (Muller, 2005)

Freuder and Quinn (Freuder \& Quinn, 1985) proposed a similar algorithm that instantiates variable that participates in most constraints. However, his approach is slightly different. The algorithm looks for a stable set in the constraint graph. A stable set of variables is a set of variables with no direct constraint among themselves. Freuder and Quinn believe that the instantiation of these variables affect the size of the search space only additively not multiplicatively, which is often the case. A constraint graph of $n$ variables with a stable set of $m$ variables has a complexity bounded by $d^{n}-m * m d$ as opposed to $d^{n}$. However, this algorithm has the shortcoming of spending time finding the 
maximal stable set. Because finding maximal stable set is a NP-hard problem itself. Therefore, it is only practical to find a suboptimal stable set for this algorithm.

Dechter (Dechter, 1990) proposed finding a cycle cutset from the set of CSP variables. A constraint graph can be made a tree by removing the cycle cutset. The cycle cutset are the nodes which cut all the cycle in the graph. The idea is to instantiate all the nodes in the cycle cutset first. Then solve the tree-structure CSP without backtracking by achieving arc consistency. This is possible based on the theorem that says: "If a constraint graph is strongly K-consistent, and $\mathrm{K}>\mathrm{W}$ where $\mathrm{w}$ is the width of the constraint graph, then there exists a search order that is backtrack free. If the size of a cycle cutset is m for a CSP with $\mathrm{n}$ variables, then the CSP can be solved in $d^{m}+(n-m) * d^{2}$ steps.

(Nadel, 1989) proposed ordering the variables by counting the number of accepted pairs in each constraint. Based on the fail-first principle, the variables with the least number of accepted pairs are selected first in the ordering. Everything else being equal, variables with fewer acceptable values are likely to fail first.

\subsubsection{Dynamic Variable Ordering}

As with the case of static variable ordering, many dynamic variable ordering heuristics have been proposed over the years. And most of these heuristics also use domain size and degree of variable as the main criterion to order the variables. However, many of the dynamic variable ordering heuristics adapted the technique of look-ahead scheme such as forward checking or MAC together with the fail-first principle. Because of constraint propagation, the domain size of the variables keep changing after forward checking, 
therefore SDF is one of the most favorite heuristic used in the research community. In this section, I will present a few of these heuristics.

(Frost \& Dechter, 1994) proposed a DVO that select a variable with the highest degree to start search if the initial domain size of the variables are the same. After the first instantiation, as a result of forward checking, the variables will have different domain size and the strategy of 'smallest domain first' is followed.

(Brelaz, 1979) proposed a DVO used for graph coloring problem which can also be applied to CSPs in general. This heuristic is similar to the one proposed by (Frost \& Dechter, 1994). It selects variables with the smallest domain first and break ties by using highest future degree of the variable. The highest future degree is the variable that involves the most constraint with the not yet instantiated variable.

Bessiere and Regin (Bessiere \& Regin, 1996) proposed a heuristic that combines both the domain size and degree of variables. The heuristic selects variables with the smallest ratio of domain size to degree to instantiate first. They believe using the ratio is better than using only the domain size or degree of variables. They were able to show that SVO using only degree to do the ordering does not perform well in the problem with very dense constraint. The heuristic degenerates to lexicographical ordering. Degree only SVO does well in problem with sparse constraints. On the other hand, SVO using domain size performs much better when the constraints are dense. Based on this observation, they believe using ratio of domain size over degree in ordering variable is better in general.

(Smith \& Grant, 1997) proposed a heuristic that is a variant to (Bessiere \& Regin, 1996). They proposed using the ratio of current domain size and future degree of variable for 
ordering. The assumption in (Bessiere \& Regin, 1996) using SDF and fail-first principle is that the probability of assigning a value that causes domain wipe out or fail is the same. However, (Smith \& Grant, 1997) argue that this may not necessary be true. Other factors such as the degree of the variable may affect the probability to fail also.

\subsection{Value Ordering Heuristics}

After selecting a variable to instantiate, there may be more than one value from the domain of the variable to choose from. The order of how these values are chosen from the domain may have a very substantial impact on the efficiency of the search. Because much of the search efforts are wasted on searching branches of the tree which do not lead to a solution when solving a hard problem in CSP. The reasoning behind the importance of value ordering is very simple. If a CSP has a solution and if a correct choice of value is chosen every time, then a solution can be found without even backtracking. This is because different value represents different branching of the node in the search tree. If the branch that leads to the solution is travelled first then no backtracking is required. The commonly applied heuristic called 'Succeed First' principle in value ordering heuristic is based on this reasoning. We always want to choose a value that can lead us quickly to the solution. As with variable ordering, the choice of a value can be made before the search begins (Static Value Ordering) or dynamically after the search begins (Dynamic Value Ordering).

Fewer heuristics for value ordering have been proposed as compared to variable ordering. This is because it is a general belief in research community that the value ordering does not make any difference if all the solutions to the constraint problem are to be found or there is no solution to the constraint problem can be found (Frost \& Dechter, 1995). This 
is because if all the solutions are required or no solution can be found in the constraint problem, the whole tree will have to be searched to find out. Every value in the domain of variables will have to be instantiated and the order doesn't matter. Most heuristics proposed in the research community are dynamic in nature. There is really no cheap heuristic to dynamically order the value. Value ordering heuristics are also very problemspecific. There is really no generic value ordering heuristic.

\subsubsection{Static Value Ordering}

(Mehta \& van Dongen, 2005) propose a static value ordering. The idea is to assign a weight to every value in the domain of every variable before the search begins. The values are sorted in decreasing order by their weights. The order remains unchanged during the search. The advantage is that no overhead is incurred to select a value after the variable is selected. The weight of the value is calculated based on the number of supports a value has if it is chosen to instantiate a variable participates in a constraint. If $\mathrm{x}$ and $\mathrm{y}$ are two variables participate in a constraint, then the support count of $a \in D(x)$, domain of $\mathrm{x}$, with respect to $\mathrm{y}$, is the number of values in $D_{a c}(y)$, the arc-consistent domain of $\mathrm{y}$, that are compatible with $a$. Let weight $[\mathrm{x}, \mathrm{a}]$ be the weight of $(\mathrm{x}, \mathrm{a})$ and let $\operatorname{scount}[\mathrm{x}, \mathrm{y}, \mathrm{a}]$ be the number of supports of $(\mathrm{x}, \mathrm{a})$ in $D_{a c}(y)$. Mehta and Van Dongen propose the following three heuristics or formulae to calculate the weight of the value.

$$
\text { weight }[x, a]=\sum_{(x, y) \in G} \operatorname{scount}[x, y, a]
$$




$$
\begin{aligned}
& \text { weight }[x, a]=\sum_{(x, y) \in G} \operatorname{scount}[x, y, a] /\left|D_{a c}(y)\right| \\
& \text { weight }[x, a]=\prod_{(x, y) \in G} \operatorname{scount}[x, y, a]
\end{aligned}
$$

In equation (1), the weight of $a$ is the sum of all the support counts of the variables participate in a constraint with $\mathrm{x}$. In equation (2), the weight of $a$ is the sum of the ratio of the sum of all support counts to arc-consistent domain of $y$ of the variables participate in a constraint with $\mathrm{x}$. In equation (3), the weight of $a$ is the product of the support counts of the variables participate in a constraint with $\mathrm{x}$. Equation (1) is essentially equivalent to the min-conflict heuristic proposed by (Frost \& Dechter, 1995). (Geelen, 1992) has proposed three heuristics for value ordering which he referred to as total-cost, cruciality and promise. These heuristics are equivalent to equations (1), (2) and (3). However, the difference is that Frost and Dechter, or Geelan's heuristics are dynamic ones. The ordering changed after the search begins.

Although the heuristics proposed do not always make the right choice of value, experimental results show that they are often better than not ordering the values of the problem. Mehta and Van Dongen also claim that ordering the values using these heuristics before the search begins may result in fewer support checks even when a problem has no solution or if search has to be done for all the solutions. 


\subsubsection{Dynamic Value Ordering}

Many dynamic value ordering heuristics use the domain sizes of future variables to determine the order of values. This is feasible because domain sizes of future variables change after constraint propagation technique such as MAC or forward checking is applied. (Frost \& Dechter, 1995) proposed a heuristic called look-ahead value (LVO) ordering with forward checking. This heuristic ranks the values in the domain of current variable based on the size of the domain of future variables after forward checking. The current variable is then instantiated with the value of the highest rank. Four heuristics functions used to determine the ranking of current domain values were proposed in (Frost \& Dechter, 1995). They are min-conflict (MC), max-domain-size (MD), weighted-maxdomain-size (WMD) and point-domain-size (PDS).

The min-conflict (MC) heuristic counts the number of incompatible value of the future variable domain after forward checking for each value in the current variable domain. The current variable domain values are than rank in the increasing order of this count.

The max-domain-size (MD) heuristic will select the value that has the largest minimum domain size in the future variables after forward checking. For example after instantiate $X_{i}=x_{1}$, the minimum domain size of future variables has 2 values, and with $X_{i}=x_{2}$, the minimum domain size of future variables has 1 value. Then $x_{1}$ will be selected.

The weighted-max-domain-size (WMD) is a refined version of MD. Because MD heuristic often creates ties situation, WMD break ties by preferring value that creates variables with fewer minimum domain size. If $x_{1}$ has 2 future variables with minimum 
domain size 2 and $x_{2}$ has 5 future variables with minimum domain size 2 , then $x_{1}$ is preferred over $x_{2}$.

The point-domain-size (PDS) heuristic gives each value in the current domain a point based on the size of the future variables domain after forward checking. For example, 8 point for each future domain size 1; 4 points for each future domain size 2 and 2 points for each future domain size 3 . The value with the smallest sum of points is selected first. All four heuristics rank the value based on the domain size of future variables. The value with the largest domain size of future variables is always preferred.

\subsection{Ordering Heuristics Based on Information Gathering and Learning}

The research community has proposed many algorithms to improve backtracking search for solving constraint satisfaction problems over the years. Basically these algorithms can be classified as either look-ahead or look-back schemes. Look-ahead schemes are designed to anticipate future conflicts before extending a partial solution during search. Usually this is done first by using constraint propagation techniques to weed out possible future conflicts to achieve some level of local consistency and then some heuristics are applied to decide which variable and value to select next to instantiate. This is called look-ahead scheme because consistency technique is applied to future variables. The most commonly known look-ahead schemes are forward checking and MAC. Look-back schemes, on the other hand, are designed to avoid future conflicts from arising again by past failures. Look-back schemes are applied whenever a dead-end is encountered during search to decide how the search should backtrack or backjump. Very often the algorithms 
also analyze the reasons for the dead-end and record them in the form of new constraints to avoid the same conflicts again.

The look-ahead and look-back schemes are usually not complementary. Base on experimental analysis, the enhancement of one scheme is often counterproductive to the other (Boussemart et al., 2004). However, in recent past new and more powerful search schemes which are a combination of the look-ahead and look-back schemes emerged for selecting variables during the search for solution. Since their emergence, these heuristics have been considered by many in the CSP research community as the most efficient general purpose heuristics for variable ordering in solving CSPs problems. This is due to the fact that these heuristics are able to identify the hard part of the problem early in the search. In the past, most heuristics, including the look-ahead and look-back schemes, use only initial state or current state of the search information to select variable. However, these newer heuristics are able to exploit also previous state of search information to guide search towards the hard parts of the CSP problem early in the search to reduce thrashing. In general, these newer heuristics work by gathering information during search to guide subsequent decision to select variable. Specifically, these heuristics gather and record information about failures in the form of constraint weight during search. This is why these heuristics are also often called conflict-directed variable ordering heuristic. The constraint weight is incremented whenever the search encounters a dead-end during constraint propagation. Each variable has a weight in these heuristics. The weight of the variable is the sum of the weights of the constraints attached to the variable. At each variable selection point, these heuristics will select a variable that has the largest weight sum. This conflict-directed approach for weighing constraint is based on the belief that 
some constraints are more important than others because they represent hard parts of the problem. Variables attached to the constraints that frequently involved in the dead-end should be instantiated early in the search to avoid thrashing and reduce the search space according to fail-first principle (Grimes \& Wallace, 2007). Extensive experiment has been done in (Boussemart et al, 2004) to show that gathering information about failure is important to circumscribe inconsistent or hard parts of CSP instances and reduce search efforts. Many researches have been done with success based on this conflict-directed approach for information gathering and weighing constraints in recent years. Among these researches are Boussemart et al.'s dynamic variable ordering heuristic which gathering information from failure to weigh constraints during consistency propagation, also called weighted degree heuristic (wdeg) and the variants of it in (Grimes \& Wallace, 2007). Boussemart et al.'s heuristic assigns a value of 1 to each constraint before the search begins. Constraint weight is incremented by 1 each time it causes a domain wipeout. At each selection point variable is then selected based on the total weight of the constraints it is attached to. The variable with the largest total weight will be selected.

The potential limitation of Boussemart et al.'s heuristic in weighing constraint is that for the first few variables selection the heuristic does not have enough information to make the best selection. The heuristic only benefit the selection at the lower level of the tree. However, the first few variables may have huge impact on the size of the search tree. Also as search goes on it may never return to those first few variables. This shortcoming of Boussemart et al.'s heuristic is remedied by random probing of the search space using search restart in Learning to identify global bottlenecks in constraint satisfaction search (Grimes \& Wallace, 2007). Grimes and Wallace proposed two methods called weighted 
information gathering (WTDI) and random information gathering (RNDI) to do random probing of the search space. Both methods assign weights to the constraints as Boussemart et al.'s heuristic that is whenever the constraint causes a domain wipeout. However, the random probing is run using a fixed number of restarts before the main search starts. Each restart is run until a cutoff time is reached. After the restart phase each constraint will have a weight assigned to it. Having enough information gathered from the restarts improves the selection of the first few variables when the main search starts. For RNDI variable selection is random at each selection point during the restart and weight is incremented as usual. On the final run when the main search starts variable is selected using the weighted degree heuristic, the variable with the largest weight will be selected first. For WTDI search restarts used information gathered from the previous run to make variable selection at the beginning of the next run and this is continued until the main search starts. Experiments show that RNDI performs better in general because of its ability to probe different part of the search space due to the random selection of variable during restarts. RNDI makes better early decision and provides better information to approximate global bottleneck because of its randomness. However, the disadvantage of RNDI is that it may not even be able to solve easy problems during the probing stage. WTDI on the other hand may solve problems during the restart or probing stage because of the information carried over each run. It should also be pointed out that RNDI is not expected to solve problem during restart. Both RNDI and WTDI are considered DVO because they continue to use the heuristic to weigh constraints in the main search and variable order can still change during the search. 
Two conflict driven heuristics similar to the random probing by (Grimes et al., 2007) are proposed by (Mouhoub \& Jafari, 2011) and proven to do better in general for randomly generated hard problems. The first heuristic is based on Hill Climbing local search to gather information and weigh constraints. The information gathered is used to order the variable before the main search start. The second proposed heuristic used to gather information before main search started is based on Ant Colony Optimization (ACO). In nature worker ants travel around to find food. These ants will leave a pheromone trails on the way back to the nest after they find the food source. Other ants may or may not follow the trail to the food source later. If they do follow the trail, they will also leave a pheromone trails and further strengthen the trails. Overtime, some trails will have stronger pheromone than others. At the end one specific trail to the food source is the shortest path. ACO is based on the ants in nature to build a construction graph with weight as in Figure 2-15. As in HC, weighted information gathered during the local search will be used in the main systematic search to order variables. Mouhoub and Jafari's method differs from (Grimes \& Wallace, 2007) in that information gathering stops after the main search starts. Therefore, Mouhoub and Jafari's method is considered static variable ordering. 


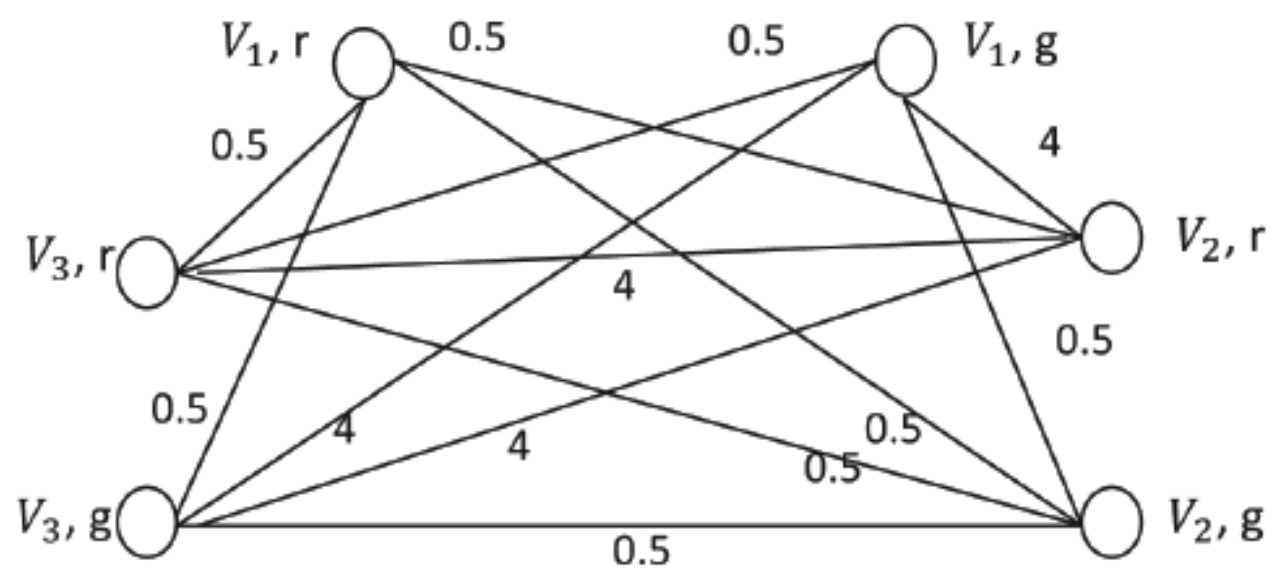

Figure 2-15 ACO construction graph (Mouhoub \& Jafari, 2011)

\subsection{Conclusion}

The aim of this chapter is to introduce some standard algorithms and techniques used for solving constraint satisfaction problems. These algorithms and techniques are important because they are center to the process of solving constraint satisfaction problems and very often they form the basis of the development of other algorithms. More importantly they are the basis of the proposed heuristics in this thesis. Some standard terminologies and concepts used in the literature are also reviewed.

I began this chapter by first giving a formal and precise definition of CSP. I demonstarted in details how a problem can be formulated as a constraint satisfaction problem by using the famous 4-Queen example. A constraint graph representation of the 4-Queen problem is also demonstarted. Having set the background for constraint satisfaction problem with precise definition and a detailed example, I continued the discussion of CSPs solving techniques. I started the discussion with the most trivial search technique, generate and test, and followed up with detailed discussion of more sophisticated backtracking and 
backjumping search techniques. The advantage of generate and test is that it is a systematic search technique. However, it cannot be used to solve large and complex problem. Backtracking search, a form of depth-first search, which is very often used for solving CSPs. It is an improvement over generate and test. However, backtracking always suffers from thrashing. Backjumping or intelligent backtracking is an improvement over backtracking because it reduces thrashing by backjumping to the cause of the dead end. Two subsections were spent to illustrate backtracking and backjumping in details because these techniques are the center to many CSP search algorithms.

I spent three subsections elaborate on constraint propagation or consistency technique because of its importance in solving CSPs. Constraint propagation is a technique used to replace CSPs by simpler ones but yet equivalent. It does so by reducing the domain size of the variables. In doing so, some branches of the search tree are pruned and the search space is reduced. Constraint propagation is very useful in the search for CSP solution if applied efficiently. It is rarely used alone in solving problems because few consistency techniques are complete. However, this is a very important technique and very few successful heuristics are not interleaved with consistency techniques in solving complex problems. Besides the fundamental concept of constraint propagation, two commonly used search algorithms that combine backtracking and consistency techniques, forward checking and MAC, are discussed in details by using the 4-Queen problem. Both of these techniques are look-ahead scheme. These two search algorithms show us how backtracking interleaved with consistency techniques are superior to either one of them alone. Forward checking prunes the search space by performing arc consistency to the future connected variables. Any value from the future variables domain that is conflicted 
with the current assignment is removed and this is the reason why it is called look-ahead scheme. Because all future assignments are guaranteed to be consistent with the past assignments. Therefore no checking with the past assignments is necessary. By performing this limited arc consistency, a future variable domain may be wiped out. If a domain wipe out occurs, it is immediately known that a deadend is encountered. This will allow the search tree that leads to the deadend to be pruned earlier. Forward checking is therefore more superior than simple backtracking. However, it should be noted that forward checking does more work at each step of the search during instantiation. MAC is an extension of forward checking. MAC does even more checking by enforcing arc consistency among all future connected variables starts from current variable. The advantage is that more deadend will be ecountered and more branches of the tree that leads to the deadend will be pruned earlier. However, even more work is required than forward checking at each step of the search. In some cases MAC may be even more expensive than doing simple backtracking.

This chapter ends with a very detailed discussion of variable and value ordering in the last three sections. Many experiments and analysis by researchers have shown that variable ordering can have a very substantial impact on the size of the search space. Variable ordering can be static or dynamic. Static variable ordering specifies the variable order before the search begins. Dynamic variable ordering selects the next variable during the search based on the current state of the search. Many heuristics have been proposed for variable ordering. Most of them are center around the fail-first principle. This principle selects next variable that is most likely to fail first. The reason is that if the current partial solution is going to fail, it is better to let it fail sooner rather than later. 
Static variable ordering (SVO) deals with the character or structure of the problem to try to come up with a good ordering. Dynamic variable ordering (DVO) deals with the search behavior or current search state to select next variable. Therefore the order of the variables may be different from one branch of the tree to the other. Some commonly used variable ordering heuristics such as dom, deg, dom/deg, ddeg, dom/ddeg, Floyd-Warshall algorithm, cycle cutset and least number of accepted pairs are introduced. After selecting a variable to instantiate, there may be more than one value from the domain of the variable to choose from. The order of how these values are chosen from the domain may have a very substantial impact on the efficiency of the search. Value ordering heuristics are based on the succeed-first principle. If a correct value is chosen every time, then a solution can be found without even backtracking. Very often much of the search efforts are wasted on searching branches of the tree that do not lead to a solution. Two value ordering heuristics proposed by (Mehta \& van Dongen, 2005) and (Frost \& Dechter, 1995) are discussed in details in this chapter. Fewer value ordering heuristics are proposed than variable ordering by the research community. The belief is that value ordering does not make any difference if all the solutions to the constraint problem are to be found or there is no solution to the problem. The whole tree will have to be searched to find out. I end the discussion of variable and value ordering heuristics by introducing a powerful search scheme based on information gathering and learning from the recent past. This search scheme is a combination of the look-ahead and look-back schemes. It has been considered by many in the research community as the most efficient general purpose heuristics for variable ordering in solving CSPs problems. This is due to the fact that these heuristics are able to identify the hard part of the problem early in the search. 
This chapter has demonstrated that much progress has been made in solving constraint satisfaction problems in the last few decades. The challenge of this thesis is how to build and continue to progress from heuristics that has been proven to be successful. 


\section{Chapter 3}

\section{Proposed Heuristics}

In this chapter I will describe the proposed dynamic variable ordering heuristic and dynamic value ordering heuristic in this thesis. The proposed dynamic variable ordering heuristic is a variant of the weighted-degree heuristic by (Boussemart et al., 2004) discussed in the background chapter. The dynamic value ordering heuristic I propose uses similar strategy to gather information and assigns weight to the constraints. Specifically, the proposed heuristics explore the possibility of using information of conflict and support count gathered during constraint propagation.

\subsection{The Proposed Variable Ordering Heuristic}

In this section I will describe the proposed dynamic variable ordering heuristic with details. In the background chapter, I have discussed the weighted-degree heuristic first proposed by (Boussemart et at., 2004) and a few variants of it by (Grimes \& Wallace, 2007; Mouhoub \& Jafari, 2011). The proposed heuristic is similar to Boussemart et al.'s method in that both use MAC as solving method during successive revision to gather information. While the variants of the original Boussemart et al.'s heuristic using restart to gather information to improve search for the first few variables early in the search, my heuristic does not use restart for random probing. Random probing using restart is certainly an elegant idea to improve search. However, there are times that we may not have the luxury to do random probing with restart. On the other hand, the importance of good selections for the first few variables is also cannot be denied as discussed in the 
background section. Therefore, my proposed heuristic begins by doing preprocessing using MAC AC-3 to gather the initial weight information. During preprocessing to maintain arc consistency, at each revision of a variable and the constraints it involves, the heuristic counts the number of conflicts and supports of the variable based on the remaining domain values of the revised variable after revision. The ratio of conflict to support counts is used as constraint weight. After the preprocessing, each constraint will have an initial weight of this ratio. The higher the ratio implies the tighter the constraint. The tighter the constraint the more likely it will cause a dead-end in future. The weight of the variable is the sum of this ratio as constraint weights attached to it. Preprocessing also has an advantage of detecting an inconsistent problem if domain wipeout occurred before search begins. After the preprocessing phase, the variable with the largest weight sum will be selected to start the search. The heuristic continues to gather information in the same way as preprocessing phase. That is at each revision point during MAC, it continues to count the conflicts and supports and use the ratio as the constraint weight. If a domain wipeout occurs during the revision, the ratio or weight of the constraint is equal to the domain size of that variable. The constraint weight information based on ratio gathered is stored for each level of the tree. Because of the extra overhead of counting the conflicts and supports for each variable, the proposed heuristic is only applied to earlier level of the search. I suggest that the counting stops after the search reach one tenth level of the tree depth.

To better explain how the proposed heuristic counts conflicts and supports, I will give an illustration in Example 1 below. The implementation of the proposed heuristic embedded 
in the revise method and MAC AC3 are shown in the pseudo code in Figure 3-1and Figure 3-2.

\section{Example 1:}

Consider a $\operatorname{CSP}(\mathrm{X}, \mathrm{D}, \mathrm{C})$ with $\mathrm{X}=\left\{x_{i}, x_{j}, x_{k}\right\}$ all having the same domain $\mathrm{D}=\{\mathrm{a}, \mathrm{b}, \mathrm{c}\}$ and $\mathrm{C}=\left\{c_{i j}, c_{i k}\right\}$. Assuming that MAC is used to solve the CSP and at some point during the search, revise method is called for the revision of variable $x_{i}$ against $c_{i j}$. That is to check if $D\left(x_{i}\right)$, the domain of variable $x_{i}$ has any support in $D\left(x_{j}\right)$, the domain of variable $x_{j}$. Any value in $D\left(x_{i}\right)$ with no support in $D\left(x_{j}\right)$ will be removed by the constraint $c_{i j}$. Assume that the constraint $c_{i j}$ has a conflict set of $c_{i j}=\{(\mathrm{a}, \mathrm{a}),(\mathrm{a}, \mathrm{b}),(\mathrm{a}, \mathrm{c}),(\mathrm{b}, \mathrm{a}),(\mathrm{b}, \mathrm{b}),(\mathrm{c}, \mathrm{a})\}$. As a result of revision, the value $\{$ a $\}$ is removed from $D\left(x_{i}\right)$, because it has no support. $D\left(x_{i}\right)$ has $\{\mathrm{b}, \mathrm{c}\}$ as remaining values now. The heuristic will now be counting the number of supports and conflicts the remaining set $\{b, c\}$ has. After enumeration $\{b, c\}$ has three supports as $\{(b, c),(c, b),(c, c)\}$ and three conflicts as $\{(b, a),(b, b),(c, a)\}$. Therefore the ratio or weight in this case for constraint $c_{i j}$ is $3 / 3$. The weight $c_{i k}$ is calculated in the similar fashion after the revision of the edge $x_{i}$ and $x_{k}$.

I would like to point out my proposed heuristic differs from both (Boussemart et al., 2004) and the variants of it in the following ways:

i) The variants of the original weighted degree procedure by Boussemart et al. in using restart to do random probing to gathered information. My proposed heuristic begins by doing preprocessing using MAC AC-3 to transform the CSP to its arc-consistent equivalent and gather the initial weight information at the same time. 
ii) Both the Boussemart et al.'s heuristic and its variants gather information about failure or domain wipe out during search to weigh constraint. My proposed heuristic gathers information about failure as well as the tightness of the constraint in the form of the ratio of conflict to support count.

iii) My proposed heuristic also differs from theirs in that the information gathering stop when the search reaches one tenth of the tree depth. The remaining search continues to use the information gathered until that point of the tree depth. 
Procedure revise $\left(X_{i}, X_{j}\right.$, domain, total $)$

Input: $D\left(X_{i}\right), D\left(X_{j}\right)$, variables $X_{i}$ and $X_{j}$.

Output: $D\left(X_{i}\right)$ such that $X_{i}$ is arc consistent wrt $X_{j}$.

total conflict count for all values in the arc consistent $D\left(X_{i}\right)$ wrt $X_{j}$ total support count for all values in the arc consistent $D\left(X_{i}\right)$ wrt $X_{j}$

begin

for each $a \in D\left(X_{i}\right)$ do

for each $v$ in $D\left(X_{j}\right)$ do

if $(a, v)$ is consistent then supportCount:=supportCount +1

endfor

if supportCount $=\mathbf{0}$ then delete $a$ from $D\left(X_{i}\right)$

conflictCount $=$ DomainSize $\left(D\left(X_{j}\right)\right)-$ supportCount

endfor

if (domain wipeout) then

set total.conflict and total.support of $X_{i}$ wrt $X_{j}$

return flag=domain wipeout

if (delete) then

set total.conflict and total.support of $X_{i}$ wrt $X_{j}$

return flag=delete

if(no delete) then

return flag $=$ no delete

end;

Figure 3-1 Pseudo code of the modified revise method 
Procedure Mac AC3(domain, tightness[][])

Input: domain of variables, tightness matrix, arc list.

Output: tightness[][] of arcs, dwo[][] count(global), arc consistent domain

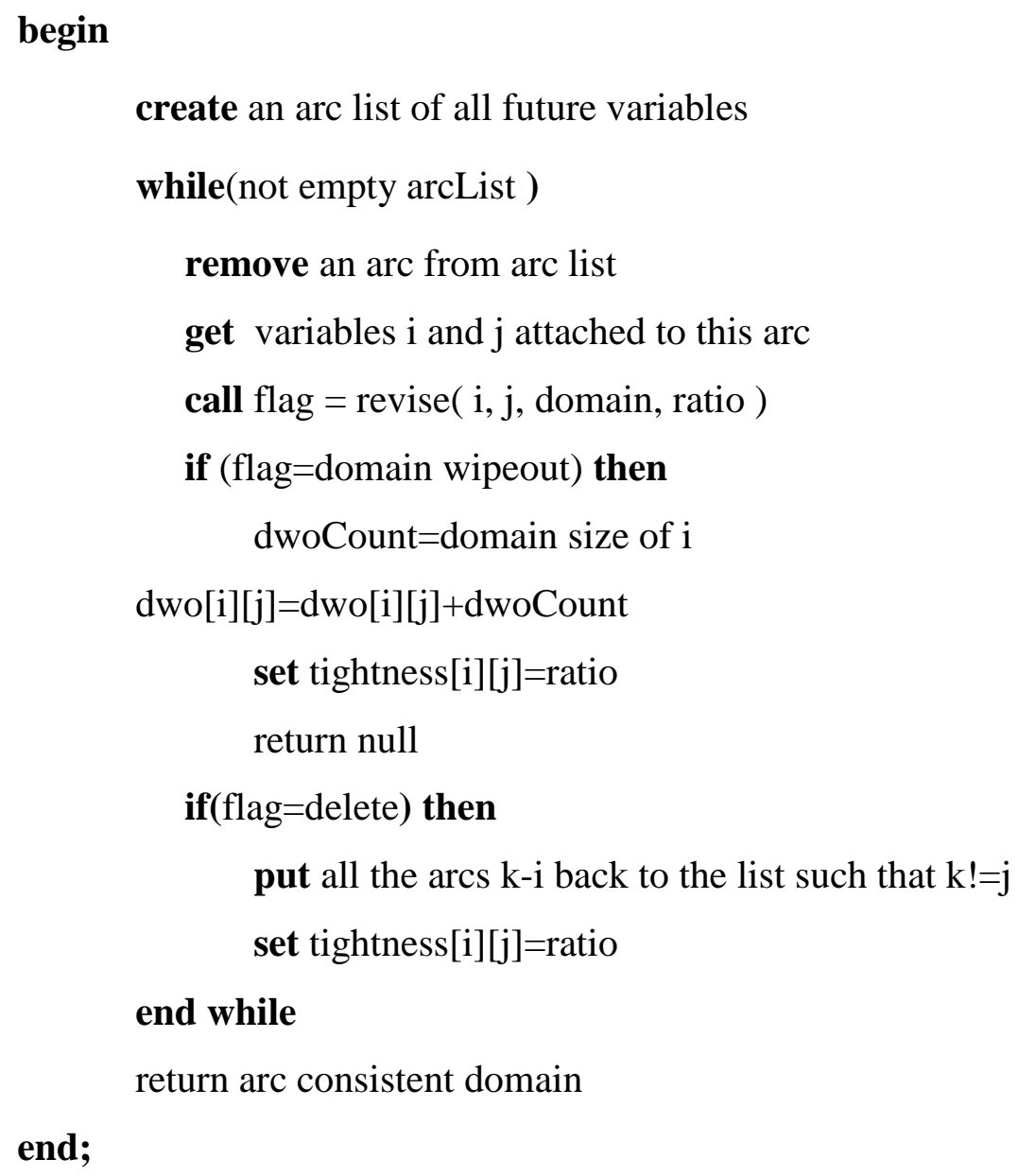

Figure 3-2 Pseudo code of the modified MAC AC3 method 


\subsection{The Proposed Value Ordering Heuristic}

In this section I will describe the proposed dynamic value ordering heuristic with details. In the background chapter, I have discussed a few value-ordering heuristics proposed in the research community. Among them are the look ahead value ordering heuristic proposed by Frost and Dechter in (Frost \& Dechter, 1995), and the static value ordering heuristic by Mehta and Van Dongen in (Mehta \& van Dongen, 2005). As discussed in the background chapter, value ordering heuristics are less often proposed in the research community because of the belief that if the CSP has no solution or all solutions have to be found then the order of the values does not matter. However it is also true that if the CSP has very few solutions then much of the time may be spent searching the branches of the tree that only lead to dead-end. Therefore, proper application of value ordering technique with variable ordering technique may have positive impact on search efficiency. This is especially true for large and hard problems as shown in (Frost \& Dechter, 1995).

The heuristic that I propose for value ordering is similar to the heuristic that I propose for variable ordering in the previous section in that it also gathers information on conflicts and supports during search and use the ratio as weight. However, this time the weight is assigned to values in the domain of the current variable and information is gathered during the forward checking phase. Each value has a weight and this weight is used to rank the values. This weight is a measure of the effect or influence the value has on the domain of the future connected variables. This influence is measured by the constraint it imposes on the domain of future connected variables using the ratio of conflicts to supports. The heuristic starts by doing a forward checking for each value in the domain of 
current variable. During the forward checking phase, each value is tentatively instantiated and checked for the number of conflicts and supports it has in the domain of the future connected variables. The inconsistent values in the future variables domain are removed. The ratio of conflicts to supports is then calculated for each future connected variable. The weight of the value is the sum of these ratios. The value with the lowest weight will be selected first to instantiate the variable. The new domains of future connected variables are also cached for each value. These domains will be used when the value is selected for instantiation in future when backtracking happens.

To better explain how the proposed heuristic computes the weight for each value, I will give an illustration in example 2 and define a formula in Example 3 below. The implementation of the proposed heuristic embedded in the revise method and MAC AC3 are shown in the pseudo code in Figure 3-3

\section{Example 2:}

Consider a $\operatorname{CSP}(X, D, C)$ with $\mathrm{X}=\left\{x_{i}, x_{j}, x_{k}\right\}$ all having the same domain $\mathrm{D}=\{\mathrm{a}, \mathrm{b}, \mathrm{c}, \mathrm{d}, \mathrm{e}\}$ and $\mathrm{C}=\left\{c_{i j}, c_{i k}\right\}$. Assume that $x_{i}$ is instantiated with $x_{i}=a$ and FC is called to do forward checking and eliminate the conflicting values in the domains of future connected variables. Also assume that the constraint $c_{i j}$ has a conflict set of $c_{i j}=\{(\mathrm{a}, \mathrm{b}),(\mathrm{a}, \mathrm{c}),(\mathrm{a}, \mathrm{d})\}$. As a result of forward checking, three values $\{b, c, d\}$ are removed from the domain of $\mathrm{D}\left(x_{j}\right)$ and the remaining values $\{\mathrm{a}, \mathrm{e}\}$ are support for $x_{i}=a$. Here the conflict count is 3 and support count is 2 for $x_{i}=a$. The weight for the edge of $x_{i}$ and $x_{k}$ is calculated in the similar way. Assume that the constraint $c_{i k}$ has a conflict set of $c_{i k}=\{(\mathrm{a}, \mathrm{a})\}$. Again, as a result of forward checking, the value $\{a\}$ conflict with $x_{i}=a$ are removed from the 
domain of $\mathrm{D}\left(x_{k}\right)$. The remaining values $\{\mathrm{b}, \mathrm{c}, \mathrm{d}, \mathrm{e}\}$ are support for $x_{i}=a$. Here the conflict count is 1 and support count is 4 for $x_{i}=a$. In this CSP, for the instantiation of $x_{i}=a$, the total conflict count is $3+1=4$. The total support count is $2+4=6$. Therefore the ratio of conflict to support count for value $x_{i}=a$ is $4 / 6$. The weight for value $x_{i}=a$ is 4/6. All other values in the domain of $x_{j}, \mathrm{D}\left(x_{j}\right)$, are calculated in the same way. The value with the smallest ratio will be selected first for instantiation.

\section{Example 3:}

Let $G$ be the constraint graph, $X$ be the current variable and $Y$ denote a future connected variable of $X$ and let weight $[X, a]$ denote the weight of a value $a \in D(X)$, the domain of current variable $X$. Also let supportCount $[X, Y, a]$ denote the support count of $a \in$ $D(X)$ with respect to a future connected variable $Y$ and let conflictCount $[X, Y, a]$ denote the conflict count of $a \in D(X)$ with respect to a future connected variable $Y$.

Then, weight $[X, a]=\sum_{(X, Y) \in G}$ conflictCount $[X, Y, a] / \operatorname{supportCount}[X, Y, a]$

The look-ahead value (LVO) ordering heuristics proposed by (Frost \& Dechter, 1995) used the similar forward checking style to collect information and rank values. However, the min-conflict heuristic proposed by Frost and Dechter used only conflicts as the measure of influence. My proposed heuristic takes into account both conflicts and supports as a measure. This constitutes the major difference between my heuristic and theirs. (Mehta \& van Dongen, 2005) also proposed a similar heuristic to rank values. However their heuristic used only support count to rank value and the ranking is done 
statically before the search starts. My heuristic ranks the value dynamically during search. 


\section{Procedure forwardChecking( ) \\ Input: current variable, $X_{\text {cur }}$.}

Output: a sorted array of conflict to support ratio of each value in $D\left(X_{\text {cur }}\right)$.

begin

for each $a \in D\left(X_{\text {cur }}\right)$ do

for each $X_{i}$, future connected variables of $X_{\text {cur }}$ do

if $\exists b \in D\left(X_{i}\right)$ such that $(\mathrm{a}, \mathrm{b})$ is consistent then supportCount=supportCount +1

if $\exists b \in D\left(X_{i}\right)$ such that $(\mathbf{a}, \mathbf{b})$ is inconsistent then remove $b$ from $D\left(X_{i}\right)$ conflictCount=conflictCount +1

endif

endfor

if any $D\left(X_{i}\right)$ becomes empty then remove $a$ from $X_{\text {cur }}$ else call MAC-AC3 to maintain arc consistency

if domain wipeout occurred during MAC-AC3 then remove $a$ from $X_{\text {cur }}$ continue to next $a$

else save the conflict to support ratio for value $a$ in the array endfor sort the array of conflict to support ratio for values $a$ in ascending order return the sorted array

end;

Figure 3-3 Pseudo code of the modified forward checking method 


\subsection{Conclusion}

In this chapter I have presented two heuristics based on conflict-directed approach with weighted degree in details. One for variable selection and one for value selection. Both proposed heuristics are dyamic in nature, that is variable or value selection is done dynamically during search. The proposed heuristics are able to identify hard parts of the problems and avoid thrashing by pruning deadend subtrees early in the search. The proposed heuristics work by gathering information about the search space before and during the search to guide subsequent variable or value selection. Specifically, the proposed heuristics gather information about failures and measure the constraint tightness by conflict to support count ratio before and during search. These informations are then recorded in the form of constraint weight. The variable or value selection is based on these constraint weights. The main contribution of my proposed heuristics is the exploitation of conflict to support count ratio to identify the hard part of the problem.

I have also demonstrated in this chapter how the proposed heuristics calculate the conflict to support count ratio by using descriptive examples and pseudo codes of the algorithms. The pseudo codes include the modified forward checking method, the modified revise method and the modified MAC-AC3 method. The pseudo code of modified forward checking method describes clearly how value selection is done by using the conflict to support count ratio. I have also pointed out the differences between my proposed heuristics and similar ones in the research community. 


\section{Chapter 4}

\section{Experimentation}

An experimental study has been conducted to evaluate the performance of the proposed variable and value ordering heuristics. In this section I will report and analyze the results of the experimental study. In the following subsections, I will first lay out the experimentation environment and evaluation criteria to give a clear picture of how the environment is set up to conduct the experiment and how the performance is evaluated. I will then give an overview of the CSP instances used in the experimental study and analyze the results obtained from the experiment.

\subsection{Experimentation Environment}

The experimental study is conducted on the Intel core i5 $\mathrm{PC}$ with $2.5 \mathrm{GHz}$ processor and 4GB memory running Windows 7 operating system. All implementations of the methods and algorithms are written in Java programming language. The CSP instances used in the experiment are taken from the renowned website http://www.cril.univartois.fr/ lecoutre/benchmarks.html. These CSP instances range from the real world application to pure random instances. I will elaborate more on these instances in the subsection below. All these CSP instances are represented in XML format. My programs will first parse the XML file to read in the instance and store them in the appropriate data structure. I have used three packages, javax.xml.parsers, org.w3c.dom and org.xml.sax for parsing the XML file. Only a total of seven classes and interfaces are used from these three packages. The classes and interfaces used are DocumentBuilder, 
DocumentBuilderFactory, ParserConfigurationException, Document, Element, NodeList and SAXException. DocumentBuilderFactory class is used to get the DocumentBuilder. DocumentBuilder class is used to parse the XML file into DOM object in the memory. Document interface is a representation of XML file. Element interface is a representation of elements in the XML file. The method getDocumentElement is used to get the elements in the XML file. To get elements within an element, the getElementsByTagName is used. To get attributes of element, getAttribute method is used. NodeList interface is used to store the elements within an element returned by getElementsByTagName. The contents of the element is extracted using getTextContent method. Both ParserConfigurationException and SAXException are checked exceptions required during the parsing of the XML file.

I have chosen to compare the results obtained using my proposed heuristic and dom/ddeg, the ratio of smallest domain size to the dynamic degree. The reason for using dom/ddeg is because it is a very efficient heuristic for many difficult problems and it is also easy to implement. Both the proposed heuristic and dom/ddeg are implemented together with backtracking and MAC-AC3. For the experiment, time limit for all problems is 1200 seconds. The program will be terminated after 1200 seconds. It is assumed that if the search does not complete within this time limit, the problem may not be able to solve by the algorithm or it may take a much longer time to solve it.

\subsection{Evaluation Criteria}

The measurements of the performance of the algorithms are collected based on three statistics: CPU time, number of constraint checks and visited nodes. These are commonly used statistics for measuring and analyzing performances of the algorithms. 
CPU time is the most important statistics of the three measurements of performance. All constraint satisfaction problem research goals are to find algorithms that can reduce the search time for solutions. However, CPU time could be very biased as a result of the environment that runs the program and the implementation of the program. Two CPU time for running two different algorithms implemented by two different programmers running on two different machines may not be comparable at all. Even if the programs were run on the same machine it may still not comparable because different implementations of the same algorithm can have significantly different CPU time. It is therefore very challenging to measure CPU time without biases. To minimize the biases that might arise, all measurements of CPU time taken are run on the same computer based on the same program design. Different methods may implement different algorithms, but the same data structures and design are used for all algorithms. I have also made an effort to use the same codes for different programs where ever possible. To futher reduce the biases, the average of the results are used after 10 runs of the same algorithm for the same instance.

Constraint checking is another important measure of algorithm performance. Constraint checking is a test for constraint violation when a variable is assigned a value. When a variable $\mathrm{x}$ is assigned a value, this assignment is checked against values in the domain of variables that involve in the constraint with $\mathrm{x}$. The constraint checking is counted as followed: when a value of a variable $\mathrm{x}$ is checked against a value of variable $\mathrm{y}$, this is counted as one constraint check. The number of constraint checks is the cost of the work done searching for solution by a CSP algorithm. This number is a good criterion for 
comparing different algorithms because it is machine independent. In general, less constraint checks means better performance.

The number of nodes visited during search is also a good measure of performance. Each instantiation is counted as one node visited. The number of visited nodes is a measure of the size of the search space explored during search. This number gives us an idea of how many assignments that did not lead to a solution. In general, smaller number of visited nodes means better performance. The number of visited nodes is also a good criterion for comparing different algorithms because it is machine independent. We can also calculate the amount of work done per assignment for each algorithm by taking the ratio of the number of constraint checks to the visited nodes. In general, the smaller the ratio the lesser is the cost per assignment or instantiation.

I have also included the number of backtracking in my experimentation for each algorithm. Fewer backtracking also means fewer visited nodes. The number of backtrackings cannot be used to measure the size of the search space explored. However, the number of backtrackings is a good indicator of whether the fail-first principle is implemented effectively. More backtrackings happen during search is an indication that a lot of thrashing and bad selection of variable. Also, comparing the number of backtrackings with the number of visited nodes is one way to identify the correctness of my program.

\subsection{CSP Instances and Experimental Evaluation}

This section provides an overview of the CSP instances used to evaluate the proposed heuristic in the experimental study. I have used a wide range of CSP instances that 
include instances from real world applications, instances following a regular pattern and involving a random generation, academic instances which do not involve any random generation, random instances containing a small structure and pure random instances. The test instances are taken from Lecoutre's website at http://www.cril.univartois.fr/ lecoutre/benchmarks.html. Many of the well-known benchmark instances can be found at this website. Some of these benchmarks have been used in various international CSP competitions and renowned research papers. These benchmarks are arranged from the top, real world instances, to the bottom, containing less and less structure, to pure random instances:

- REAL: instances from real world applications,

- PATT: patterned instances following a regular pattern and involving a random generation,

- ACAD: instances from the academia and which do not involve any random generation,

- QRND: quasi-random instances containing a small structure,

- RAND: pure random instances.

In the following subsections, I will give a brief introduction of the CSP instnaces used in the experimental study and followed by the analysis of the experimental results.

\subsubsection{Real World Instances: The Driverlog Problem}

For real world problems I have chosen the Driverlog problem for testing. The Driverlog problem is a logistic planning problem. Each problem involves four types of objects: locations, trucks, drivers and packages. Locations are connected by roads and paths. 
Packages have to be transported by truck and driven by driver. The goal of Driverlog is how to move a subset of drivers, trucks and packages to certain locations.

The results in table 4-1 below show that dom/wdeg heuristic is better than dom/ddeg in general. The Driverlog instances in the table is arranged from the top to bottom in increasing difficulty. At the top of the table is instance driverlogw-01c-sat_ext, having 3 domains of different sizes with maximum domain size of 4 values, 71 variables, 14 relations and 217 constraints. At the bottom of the table is instance driverlogw-09sat_ext, having 9 domains of different sizes with maximum domain size of 12 values, 650 variables, 1639 relations and 17447 constraints. Not only do the instances become larger towards the bottom of table, but their relations also have become more varied in terms of constraint tightness. Different relations have very different constraint tightness. The proposed heuristic performs better in terms of CPU time as compared to dom/ddeg towards the bottom of the table in general. As can be seen from the results for problem instances driverlogw-08cc-sat_ext and driverlogw-08c-sat_ext where the difference in CPU time is significant. The reason that the proposed heuristic only outperforms dom/ddeg significantly towards the bottom of the table in terms of CPU time is because the extra overhead of calculating the constraint weights by conflict to support ratio does not pay off with smaller problems. One of the reason that dom/ddeg performs worse is because many variables with higher degree may be attached to constraints of lower tightness. The selection of variable based on degree is not accurate for instances with varying degree of tightness compared to the proposed heuristic. The variables with tightest constraints may not be selected first and this lead to a larger search tree being traversed. In terms of the number of visited nodes and constraint checks, the proposed 
heuristic also outperforms dom/ddeg. Based on the number of visited nodes and constraint checks, the proposed heuristic has definitely made a good choice in variable selection and successfully prune off many subtrees.

Table 4-1 Results for Driverlog instances

\begin{tabular}{|c|c|c|c|}
\hline Instances & Measurements & dom/wdeg & dom/ddeg \\
\hline $\begin{array}{c}\text { driverlogw-01c-sat_ext } \\
\text { (sat) }\end{array}$ & $\begin{array}{c}\text { cpu seconds } \\
\text { \#vn } \\
\# \text { cck }\end{array}$ & $\begin{array}{c}0.025 \\
70 \\
0.026 \mathrm{M}\end{array}$ & $\begin{array}{c}0.107 \\
72 \\
0.029 \mathrm{M}\end{array}$ \\
\hline $\begin{array}{c}\text { driverlogw-02c-sat_ext } \\
\text { (sat) }\end{array}$ & $\begin{array}{c}\text { cpu seconds } \\
\text { \#vn } \\
\# \text { cck }\end{array}$ & $\begin{array}{c}32.848 \\
1917 \\
40.400 \mathrm{M}\end{array}$ & $\begin{array}{c}32.934 \\
8452 \\
79.092 \mathrm{M}\end{array}$ \\
\hline $\begin{array}{c}\text { driverlogw-04c-sat_ext } \\
\text { (sat) }\end{array}$ & $\begin{array}{c}\text { cpu seconds } \\
\text { \#vn } \\
\# \text { cck }\end{array}$ & $\begin{array}{c}2.323 \\
351 \\
3.454 \mathrm{M}\end{array}$ & $\begin{array}{c}2.848 \\
501 \\
5.460 \mathrm{M}\end{array}$ \\
\hline $\begin{array}{c}\text { driverlogw-05c-sat_ext } \\
\text { (sat) }\end{array}$ & $\begin{array}{c}\text { cpu seconds } \\
\text { \#vn } \\
\# \text { cck }\end{array}$ & $\begin{array}{c}18.198 \\
603 \\
14.109 \mathrm{M}\end{array}$ & $\begin{array}{c}6.433 \\
938 \\
13.014 \mathrm{M}\end{array}$ \\
\hline $\begin{array}{c}\text { driverlogw-08cc-sat_ext } \\
\text { (sat) }\end{array}$ & $\begin{array}{c}\text { cpu seconds } \\
\# \text { vn } \\
\# \text { cck }\end{array}$ & $\begin{array}{c}66.641 \\
1481 \\
51.222 \mathrm{M}\end{array}$ & $\begin{array}{c}116.824 \\
7606 \\
177.126 \mathrm{M}\end{array}$ \\
\hline $\begin{array}{c}\text { driverlogw-08c-sat_ext } \\
\text { (sat) }\end{array}$ & $\begin{array}{c}\text { cpu seconds } \\
\text { \#vn } \\
\# \text { cck }\end{array}$ & $\begin{array}{c}80.069 \\
1689 \\
59.881 \mathrm{M}\end{array}$ & $\begin{array}{c}121.428 \\
7606 \\
177.153 \mathrm{M}\end{array}$ \\
\hline $\begin{array}{c}\text { driverlogw-09-sat_ext } \\
\text { (sat) }\end{array}$ & $\begin{array}{c}\text { cpu seconds } \\
\text { \#vn } \\
\# \text { cck }\end{array}$ & $\begin{array}{c}>1200 \\
\text { N/A } \\
\text { N/A }\end{array}$ & $\begin{array}{c}>1200 \\
\text { N/A } \\
\text { N/A }\end{array}$ \\
\hline
\end{tabular}

\subsubsection{Patterned Instances}

For patterned instances, I have used balanced Quasigroup with holes (BQWH) and Quasi completion problems for testing. These instances are very challenging benchmarks of combinatorial problems. The problems are random with structures. Random instances seldom have the structures found in the real world problem. QCP are used to bridge the gap between random instances and structured problems. The QCP problems have many practical applications in real world domain such as conflict-free wavelength routing in 
wide band optical networks, scheduling, statistical design, and error correcting codes. A Quasigroup can be viewed as an NXN multiplication table defining a Latin square. Each row and column of the table must be filled with unique integer values. The Quasigroup Completion Problem (QCP) is the problem of completing a partially filled Latin square. Quasigroup with Holes (QWH) are satisfiable instances. They are generated by starting with a complete Latin square or complete Quasigroup and removing some of the entries. $\mathrm{BQWH}$ are harder when the distribution of the holes is balanced or the number of unassigned cells is approximately the same across the different rows and columns (Gomes \& Walsh, 2006).

Table 4-2 shows the result of testing 6 instances of BQWH and QCP each. The instances in the table are arranged in increasing size of the problem from top to bottom. As can be seen from the results, the proposed heuristic performs better than dom/ddeg in general for balanced quasi group with holes (BQWH) instances for larger problems like bqwh-18141-0 and bqwh-18-141-6. However, as the results show, the proposed heuristic does not always outperform dom/ddeg very significantly for smaller and easy problems. This is because for this group of problems the domain size is not very big and the constraint tightness of the relations is low and not very different from each other to begin with. Therefore the measure of constraint weight by conflicts to supports ratio may not be cost effective for smaller problems. However, the conflict-based information from the conflict to support count ratio and domain wipeout is always accurate as can be seen from the number of visited nodes and constraint checks. 
For quasi completion problems (QCP), the proposed heuristic performs better than dom/ddeg in general as the problem gets larger and more difficult. For smaller problems the performance is about the same. The proposed heuristic significantly outperforms dom/ddeg in two instances, qcp-15-120-8 and qcp-15-120-14. One reason is because these two instances are large and difficult. However, the main reason is because most variables are having about the same degree but some variables are involved in relations that are much more constraint than others. The proposed heuristic was able to pick these variables first to instantiate and prune the search tree early in the search. This is particularly true for instance qcp-15-120-14. For this instance, the proposed heuristic is able to show that the problem has no solution in less than one second whereas dom/ddeg ran out of time limit without being able to solve the problem. The dom/ddeg heuristic keeps thrashing for selecting the wrong variables to instantiate.

The patterned instances are random with structure. The structure of the problem may dictate which heuristic works better. However, it is safe to say that for patterned instances of larger size, the proposed heuristic very often performs much better than dom/ddeg. This is because early discovery of the hard part of the problem can save a lot of search time for large problem. The extra overhead for detecting the hard part of the problem always get payoff for large instance. 
Table 4-2 Results for Quasigroup patterned instances

\begin{tabular}{|c|c|c|c|}
\hline Instances & Measurements & dom/wdeg & dom/ddeg \\
\hline $\begin{array}{c}\text { bqwh-15-106-0 } \\
\text { (sat) }\end{array}$ & $\begin{array}{c}\text { cpu seconds } \\
\# \mathrm{vn} \\
\# \mathrm{cck}\end{array}$ & $\begin{array}{c}1.161 \\
1580 \\
5.685 \mathrm{M}\end{array}$ & $\begin{array}{c}1.936 \\
4970 \\
9.791 \mathrm{M}\end{array}$ \\
\hline $\begin{array}{c}\text { bqwh-15-106-5 } \\
\text { (sat) }\end{array}$ & $\begin{array}{c}\text { cpu seconds } \\
\# \mathrm{vn} \\
\# \text { cck }\end{array}$ & $\begin{array}{c}0.135 \\
167 \\
0.387 \mathrm{M}\end{array}$ & $\begin{array}{c}0.815 \\
2110 \\
4.268 \mathrm{M}\end{array}$ \\
\hline $\begin{array}{c}\text { bqwh-15-106-9 } \\
\text { (sat) }\end{array}$ & $\begin{array}{c}\text { cpu seconds } \\
\# \text { vn } \\
\# \text { cck }\end{array}$ & $\begin{array}{c}0.662 \\
781 \\
2.664 \mathrm{M}\end{array}$ & $\begin{array}{c}3.648 \\
9143 \\
19.502 \mathrm{M} \\
\end{array}$ \\
\hline $\begin{array}{c}\text { bqwh-18-141-0 } \\
\text { (sat) }\end{array}$ & $\begin{array}{c}\text { cpu seconds } \\
\# \mathrm{vn} \\
\# \mathrm{cck} \\
\end{array}$ & $\begin{array}{c}11.161 \\
6540 \\
43.685 \mathrm{M} \\
\end{array}$ & $\begin{array}{c}377.696 \\
584461 \\
1688.343 \mathrm{M} \\
\end{array}$ \\
\hline $\begin{array}{c}\text { bqwh-18-141-6 } \\
\text { (sat) }\end{array}$ & $\begin{array}{c}\text { cpu seconds } \\
\# \text { vn } \\
\# \text { cck } \\
\end{array}$ & $\begin{array}{c}3.55 \\
1777 \\
12.300 \mathrm{M} \\
\end{array}$ & $\begin{array}{c}132.084 \\
177525 \\
588.053 \mathrm{M} \\
\end{array}$ \\
\hline $\begin{array}{c}\text { bqwh-18-141-22 } \\
\text { (sat) }\end{array}$ & $\begin{array}{c}\text { cpu seconds } \\
\# \text { vn } \\
\# \text { cck } \\
\end{array}$ & $\begin{array}{c}0.454 \\
471 \\
1.650 \mathrm{M} \\
\end{array}$ & $\begin{array}{c}5.992 \\
11025 \\
25.173 \mathrm{M} \\
\end{array}$ \\
\hline $\begin{array}{l}\text { qcp-10-67-0 } \\
\text { (sat) }\end{array}$ & $\begin{array}{c}\text { cpu seconds } \\
\# \text { vn } \\
\# \text { cck }\end{array}$ & $\begin{array}{c}0.052 \\
99 \\
0.133 \mathrm{M} \\
\end{array}$ & $\begin{array}{c}0.058 \\
99 \\
0.254 \mathrm{M} \\
\end{array}$ \\
\hline $\begin{array}{c}\text { qcp-10-67-7 } \\
\quad \text { (sat) }\end{array}$ & $\begin{array}{c}\text { cpu seconds } \\
\# \mathrm{vn} \\
\# \text { cck }\end{array}$ & $\begin{array}{c}0.424 \\
276 \\
1.452 \mathrm{M}\end{array}$ & $\begin{array}{c}0.056 \\
144 \\
0.283 \mathrm{M}\end{array}$ \\
\hline $\begin{array}{l}\text { qcp-10-67-14 } \\
\text { (unsat) }\end{array}$ & $\begin{array}{c}\text { cpu seconds } \\
\# \mathrm{vn} \\
\# \text { cck }\end{array}$ & $\begin{array}{c}0.044 \\
9 \\
0.089 \mathrm{M}\end{array}$ & $\begin{array}{c}0.051 \\
72 \\
0.272 \mathrm{M}\end{array}$ \\
\hline $\begin{array}{c}\text { qcp-15-120-0 } \\
\text { (sat) }\end{array}$ & $\begin{array}{c}\text { cpu seconds } \\
\# \mathrm{vn} \\
\# \text { cck }\end{array}$ & $\begin{array}{c}6.725 \\
1480 \\
12.835 \mathrm{M}\end{array}$ & $\begin{array}{c}45.511 \\
73301 \\
155.419 \mathrm{M}\end{array}$ \\
\hline $\begin{array}{c}\text { qcp-15-120-8 } \\
\text { (sat) }\end{array}$ & $\begin{array}{c}\text { cpu seconds } \\
\# \mathrm{vn} \\
\# \mathrm{cck} \\
\end{array}$ & $\begin{array}{c}19.284 \\
3380 \\
38.085 \mathrm{M} \\
\end{array}$ & $\begin{array}{c}76.339 \\
108681 \\
281.100 \mathrm{M} \\
\end{array}$ \\
\hline $\begin{array}{c}\text { qcp-15-120-14 } \\
\text { (unsat) }\end{array}$ & $\begin{array}{c}\text { cpu seconds } \\
\# \mathrm{vn} \\
\# \text { cck }\end{array}$ & $\begin{array}{c}0.605 \\
50 \\
1.564 \mathrm{M}\end{array}$ & $\begin{array}{c}>1200 \\
\text { NA } \\
\text { NA }\end{array}$ \\
\hline
\end{tabular}

\subsubsection{Academic Instances}

For academic instances, I have used the Tower of Hanoi problems for testing. The Tower of Hanoi problems is to transfer an entire tower of disks from one peg to another. Given n disks, stack on one of the three pegs. The disks are initially stack with the largest one on 
the bottom to the smallest one on the top. The task is to transfer the entire stack of disks to another peg with the constraint that moving only one disk at a time and never place the larger disk onto the smaller one.

Table 4-3 below shows the results of testing 3 instances of the Tower of Hanoi problem. The difference between the proposed heuristic and dom/ddeg is not very significant. This is because the problem is very easy to solve. As can be seen from the table results, the largest problem is hanoi-7_ext, takes only 0.263 CPU seconds to solve. Hanoi instances have only three domains and two of then have only 2 values.

Table 4-3 Results for the Tower of Hanoi instances

\begin{tabular}{|c|c|c|c|}
\hline \multirow{2}{*}{$\begin{array}{c}\text { Instances } \\
\text { hanoi-5_ext } \\
\text { (sat) }\end{array}$} & Measurements & dom/wdeg & dom/ddeg \\
\cline { 2 - 4 } & cpu seconds & 0.009 & 0.027 \\
\cline { 2 - 4 } & \#vn & 30 & 30 \\
\hline \multirow{2}{*}{$\begin{array}{c}\text { hanoi-6_ext } \\
\text { (sat) }\end{array}$} & cpu seconds & 0.031 & $0.21 \mathrm{e}-4 \mathrm{M}$ \\
\cline { 2 - 4 } & \#vn & 61 & 0.015 \\
\hline \multirow{2}{*}{$\begin{array}{c}\text { hanoi-7_ext } \\
\text { (sat) }\end{array}$} & cpu seconds & $0.003 \mathrm{M}$ & $0.001 \mathrm{M}$ \\
\cline { 2 - 4 } & \#vn & 125 & 0.263 \\
\cline { 2 - 4 } & \#cck & $0.015 \mathrm{M}$ & 125 \\
\hline
\end{tabular}

\subsubsection{Quasi-Random Instances}

For Quasi-Random instances I have used the Composed, Ehi and Geometric Instances for testing. The Composed instances in Table 3 are random CSP instances such that each generated instance is composed of a main, under-constrained fragment, and some auxiliary fragments, each of which being grafted to the main one by introducing some binary constraints. Some composed instances used are not satisfiable. 
Ehi85 and ehi90 instances are two series of 3SAT instances. A 3-SAT instance is a SAT instance such that each clause contains exactly 3 literals. These are easy random 3-SAT instances embedding with a small unsatisfiable part. Therefore all instances are unsatisfiable. These instances are originated in (Bayardo \& Schrag, 1997) and have been converted into binary CSP instances using the dual method that is described in (Bacchus, 2000).

The Geometric instances are random instances proposed and generated by Rick Wallace. These random instances are generated using geometric methods as follows. Instead of using a density parameter, a distance parameter is used such that dst $\leq \sqrt{2}$. For each variable, two coordinates are chosen at random so the associated point lies in the unit square. Then for each variable pair, $(\mathrm{x}, \mathrm{y})$, if the distance between their associated points is less than or equal to distance parameter dst, the arc $(\mathrm{x}, \mathrm{y})$ is added to the constraint graph. Constraint relations are created in the same way as they are for homogeneous random CSP instances. Each instance is prefixed by geom.

The table 4-4 below shows the result of testing these instances. As can be seen from the results the proposed heuristic is superior to dom/ddeg in all but one instance. The proposed heuristic was able to solve the problem or prove that the instance has no solution within seconds whereas dom/ddeg is running beyond the time limit. This is because some parts of the Composed instances are very dense and some variables are constrained by same number of degrees of very different tightness in the graph. Because of this dom/ddeg may be reduced to dom only heuristic or backtracking. This explains why dom/ddeg is running beyond the time limit without solving the problem. However, the proposed heuristic was able to gather information and identify the hard part of the 
problem during MAC before search begins. This information gathered based on conflicts to support ratio has enable the proposed heuristic to correctly select variables that are likely leading the search to the deadend . Subtrees leading to the deadend are pruned immediately and no backtrack is even required. This is the benefit of conflict directed heuristic. The ability of finding deadend early and saving search time. Two satisfiable instances in the table, composed-25-10-20-0 and composed-25-10-20-5, both have 105 variables. The proposed heuristic is able to solve the problems with minimal effort as the number of visited nodes are 110 only. However, these two instances have caused dom/ddeg to run out of time limit. For this group of problems, dom/ddeg is often reduced to backtracking and thrashing in the hard part of the search space. The proposed heuristic also outperforms dom/ddeg for instances ehi-85-297-0_ext, ehi-85-297-56_ext and ehi90-315-1_ext. Ehi instances are all unsatisfiable because they are embedded with a small unsatisfiable part. The proposed heuristic is able to identify the unsatisfiable part early and prove that the problem has no solution whereas dom/ddeg is running out of time limit. Ehi instances are large. They consist of 297 variables, 77 relations of varying tightness and the problems have 4102 constraints. The inability of dom/ddeg to identify the deadend early causing the search to thrash and run out of time. 
Table 4-4 Results for Quasi-Random instances

\begin{tabular}{|c|c|c|c|}
\hline Instances & Measurements & dom/wdeg & dom/ddeg \\
\hline $\begin{array}{c}\text { composed-25-1-2-0 } \\
\text { (unsat) }\end{array}$ & $\begin{array}{c}\text { cpu seconds } \\
\# \text { vn } \\
\# \text { cck }\end{array}$ & $\begin{array}{c}0.015 \\
1 \\
0.034 \mathrm{M}\end{array}$ & $\begin{array}{c}>1200 \\
\text { NA } \\
\text { NA }\end{array}$ \\
\hline $\begin{array}{c}\text { composed-25-1-2-5 } \\
\text { (unsat) }\end{array}$ & $\begin{array}{c}\text { cpu seconds } \\
\text { \#vn } \\
\text { \#cck }\end{array}$ & $\begin{array}{c}0.015 \\
1 \\
0.043 \mathrm{M}\end{array}$ & $\begin{array}{c}>1200 \\
\text { NA } \\
\text { NA }\end{array}$ \\
\hline $\begin{array}{c}\text { composed-25-1-40-0 } \\
\text { (unsat) }\end{array}$ & $\begin{array}{c}\text { cpu seconds } \\
\text { \#vn } \\
\text { \#cck }\end{array}$ & $\begin{array}{c}0.015 \\
1 \\
0.038 \mathrm{M}\end{array}$ & $\begin{array}{c}>1200 \\
\text { NA } \\
\text { NA }\end{array}$ \\
\hline $\begin{array}{c}\text { composed-25-1-25-0 } \\
\text { (unsat) }\end{array}$ & $\begin{array}{c}\text { cpu seconds } \\
\text { \#vn } \\
\text { \#cck }\end{array}$ & $\begin{array}{c}0.011 \\
1 \\
0.036 \mathrm{M}\end{array}$ & $\begin{array}{c}>1200 \\
\text { NA } \\
\text { NA }\end{array}$ \\
\hline $\begin{array}{c}\text { composed-25-10-20-0 } \\
\text { (sat) }\end{array}$ & $\begin{array}{c}\text { cpu seconds } \\
\# \text { vn } \\
\# \text { cck }\end{array}$ & $\begin{array}{c}0.107 \\
110 \\
0.785 \mathrm{M}\end{array}$ & $\begin{array}{c}>1200 \\
\text { NA } \\
\text { NA }\end{array}$ \\
\hline $\begin{array}{c}\text { composed-25-10-20-5 } \\
\text { (sat) }\end{array}$ & $\begin{array}{c}\text { cpu seconds } \\
\text { \#vn } \\
\# \text { cck }\end{array}$ & $\begin{array}{c}0.102 \\
110 \\
0.749 \mathrm{M}\end{array}$ & $\begin{array}{c}>1200 \\
\text { NA } \\
\text { NA }\end{array}$ \\
\hline $\begin{array}{c}\text { composed-75-1-80-0 } \\
\text { (unsat) }\end{array}$ & $\begin{array}{c}\text { cpu seconds } \\
\text { \#vn } \\
\# \text { cck }\end{array}$ & $\begin{array}{c}0.037 \\
1 \\
0.121 \mathrm{M}\end{array}$ & $\begin{array}{c}0.027 \\
1 \\
0.107 \mathrm{M}\end{array}$ \\
\hline $\begin{array}{c}\text { composed-75-1-80-5 } \\
\text { (unsat) }\end{array}$ & $\begin{array}{c}\text { cpu seconds } \\
\# \mathrm{vn} \\
\# \mathrm{cck}\end{array}$ & $\begin{array}{c}0.037 \\
1 \\
0.137 \mathrm{M}\end{array}$ & $\begin{array}{c}>1200 \\
\text { NA } \\
\text { NA }\end{array}$ \\
\hline $\begin{array}{l}\text { ehi-85-297-0_ext } \\
\text { (unsat) }\end{array}$ & $\begin{array}{c}\text { cpu seconds } \\
\# \text { vn } \\
\# \text { cck }\end{array}$ & $\begin{array}{c}0.271 \\
6 \\
0.790 \mathrm{M}\end{array}$ & $\begin{array}{c}>1200 \\
\text { NA } \\
\text { NA }\end{array}$ \\
\hline $\begin{array}{c}\text { ehi-85-297-56_ext } \\
\text { (unsat) }\end{array}$ & $\begin{array}{c}\text { cpu seconds } \\
\text { \#vn } \\
\# \text { cck }\end{array}$ & $\begin{array}{c}0.176 \\
1 \\
0.109 \mathrm{M}\end{array}$ & $\begin{array}{c}>1200 \\
\text { NA } \\
\text { NA }\end{array}$ \\
\hline $\begin{array}{c}\text { ehi-90-315-1_ext } \\
\text { (unsat) }\end{array}$ & $\begin{array}{c}\text { cpu seconds } \\
\text { \#vn } \\
\# \text { cck }\end{array}$ & $\begin{array}{c}0.118 \\
1 \\
0.217 \mathrm{M}\end{array}$ & $\begin{array}{c}>1200 \\
\text { NA } \\
\text { NA }\end{array}$ \\
\hline $\begin{array}{c}\text { geo50-20-d4-75-1_ext } \\
\text { (sat) }\end{array}$ & $\begin{array}{c}\text { cpu seconds } \\
\text { \#vn } \\
\text { \#cck }\end{array}$ & $\begin{array}{c}242.622 \\
81262 \\
1733.405 \mathrm{M}\end{array}$ & $\begin{array}{c}368.64 \\
425369 \\
2535.033 \mathrm{M}\end{array}$ \\
\hline
\end{tabular}

\subsubsection{Random Instances}

For random instances, I have used the satisfiable SAT benchmark problems, frb series, for testing. These instances are particularly interesting as they are hard to solve. The frb series used for testing consist of 8 benchmark sets of forced satisfiable problems. These 
are hard satisfiable SAT problems directly encoded from forced satisfiable CSP instances using Model RB in (Xu, 2015). All instances are located in the region of phase transition follow Model RB. The 8 benchmark sets constitute of problem size growing from 450 Boolean variables to 1534 Boolean variables. The problems get more difficult as the number of Boolean variables increase because $\alpha, \mathrm{r}$ and constraint tightness $p$ are fixed at the phase transition. Domain size increases with the number of variables for Model RB because $d=n^{\alpha}$. The constraint tightness increases with $d$ because for each constraint Model RB selects $p d^{2}$ incompatible pairs of values.

Table 4-5 below presents the result of the experimentation conducted using the frb series. The instances in the table are arranged in increasing difficulty from top to bottom. At the top of the table, instances frb30-15 have 30 variables and all variables have 15 domain values. Towards the bottom of the table, instances frb40-19 have 40 variables and all variables have 19 domain values. As can be seen, the proposed heuristic performs better than dom/ddeg in general. However, the difference in performance is not significant until the problems gets larger towards the bottom of the table. This is because instances from Model RB have the same constraint tightness for all its relations before the search starts. Because of that dom/ddeg is more efficient earlier in the search and for smaller or easier problems. As the search goes on and gets deeper in the search space, after some variables are instantiated, more values will be eliminated by MAC. Constraint tightness of the remaining relations may change and differs significantly in terms of conflict to support count ratio. This is the time when the work done for calculating the ratio of conflicts to supports for dom/wdeg gets payoff. For some instances, if this change in constraint tightness among the relations differs very significantly, this is when the proposed 
heuristic will outperforms dom/ddeg significantly in CPU time. This is the case for instance frb35-17-5-mgd_ext in the table below, where the CPU time performance is about 40 times better. The experimental results also show that the proposed heuristic performs better than dom/ddeg in terms of visited nodes and constraint checks for all smaller and larger problems. This shows that the variable selection based on conflict to support ratio is correct. For larger problems the differences in constraint checks and visited nodes are very significant as in the instances of frb40-19-1-mgd_ext and frb40-195-mgd_ext. The difference is in 100 millions for constraint checks and over 10 thousands in visited nodes. For instance frb40-19-1-mgd_ext, the extra work done for dom/ddeg is more than 287 millions in constraint checks and 30353 in visited nodes. For instance frb40-19-5-mgd_ext, the extra work done is more than 245 millions in constraint checks and 55007 in visited nodes. As problems get even larger this extra works done can get even bigger. The results from visited nodes and constraint checks are important because they are implementation independent. Based on these results, the proposed heuristic has definitely made good selection of variables and save significant amount of work done for larger problems. 
Table 4-5 Results for forced satisfiable Random instances

\begin{tabular}{|c|c|c|c|}
\hline & & & \\
Instances & Measurements & dom/wdeg & dom/ddeg \\
\hline frb30-15-1-mgd_ext & cpu seconds & 0.213 & 0.259 \\
(sat) & $\#$ vn & 247 & 382 \\
& \#cck & $1.307 \mathrm{M}$ & $1.935 \mathrm{M}$ \\
\hline \multirow{2}{*}{ frb30-15-5-mgd_ext } & cpu seconds & 0.877 & 1.008 \\
(sat) & $\#$ \#n & 942 & 1191 \\
(sat) & \#cck & $6.335 \mathrm{M}$ & $6.821 \mathrm{M}$ \\
\hline \multirow{2}{*}{ frb35-17-1-mgd_ext } & cpu seconds & 5.833 & 7.891 \\
(sat) & \#cck & 4377 & 7933 \\
frb35-17-5-mgd_ext & cpu seconds & 1.343 & $58.428 \mathrm{M}$ \\
\hline \multirow{2}{*}{ frb40-19-1-mgd_ext } & \#vn & 1062 & 41.934 \\
(sat) & cpu secck & $8.271 \mathrm{M}$ & 41925 \\
\hline \multirow{2}{*}{ frb40-19-5-mgd_ext } & \#vn & 40.911 & $297.043 \mathrm{M}$ \\
(sat) & cpu seconds & 22509 & 74.321 \\
& \#vn & 164.369 & 52862 \\
\hline & \#cck & $1082.125 \mathrm{M}$ & $1327.581 \mathrm{M}$ \\
\hline
\end{tabular}

Table 4-6 below shows the results when both the proposed variable and value ordering heuristics are used together in the testing. For this part of the testing, I have also used the random instances generated using Model-RB, the rand series. The performance is much better when both heuristics are used together for larger and more difficult problems. This can be seen from the result of testing instances frb45-21-1-mgd_ext and rand-2-40-19443-230-fcd-11_ext. For these two instances, the performance is better in all three categories of time, number of visited nodes and constraint checks. For easy problems the extra overhead used to rank values are not cost effective. Easy problems with many acceptable values for instantiation make the value ordering heuristic expensive. It should be noted that when value ordering is used, the numbers of constraint checks and visited nodes are always more than if it is not used. The extra constraint checks and visited nodes are the overhead for ranking the values. However, when the problem gets very difficult, the constraint checks and visited nodes become much less. This is when the extra 
overhead used to rank the values gets pay off. The value ranking actually has saved support checks and reduced search space.

Table 4-6 Results when variable and value ordering heuristics are used together

\begin{tabular}{|c|c|c|c|}
\hline Instances & Measurements & dom/wdeg & $\begin{array}{l}\text { dom/wdeg with } \\
\text { value ordering }\end{array}$ \\
\hline $\begin{array}{c}\text { frb35-17-1-mgd_ext } \\
\text { (sat) }\end{array}$ & $\begin{array}{c}\text { cpu seconds } \\
\# \text { vn } \\
\# \text { cck }\end{array}$ & $\begin{array}{c}5.833 \\
4377 \\
40.060 \mathrm{M} \\
\end{array}$ & $\begin{array}{c}6.759 \\
7326 \\
69.075 \mathrm{M} \\
\end{array}$ \\
\hline $\begin{array}{c}\text { frb35-17-5-mgd_ext } \\
\text { (sat) }\end{array}$ & $\begin{array}{c}\text { cpu seconds } \\
\text { \#vn } \\
\# \text { cck }\end{array}$ & $\begin{array}{c}1.343 \\
1062 \\
8.271 \mathrm{M}\end{array}$ & $\begin{array}{c}8.983 \\
10633 \\
83.644 \mathrm{M}\end{array}$ \\
\hline $\begin{array}{c}\text { frb40-19-5-mgd_ext } \\
\text { (sat) }\end{array}$ & $\begin{array}{c}\text { cpu seconds } \\
\text { \#vn } \\
\# \text { cck } \\
\end{array}$ & $\begin{array}{c}164.369 \\
118428 \\
1082.125 \mathrm{M} \\
\end{array}$ & $\begin{array}{c}177.247 \\
169750 \\
1547.389 \mathrm{M}\end{array}$ \\
\hline $\begin{array}{c}\text { frb45-21-1-mgd_ext } \\
\text { (sat) }\end{array}$ & $\begin{array}{c}\text { cpu seconds } \\
\text { \#vn } \\
\# \text { cck } \\
\end{array}$ & $\begin{array}{c}5107.639 \\
2849545 \\
29534.969 \mathrm{M} \\
\end{array}$ & $\begin{array}{c}419.211 \\
322759 \\
3265.371 \mathrm{M} \\
\end{array}$ \\
\hline $\begin{array}{c}\text { rand-2-30-15-306-230- } \\
\text { fcd-1_ext } \\
\text { (sat) }\end{array}$ & $\begin{array}{c}\text { cpu seconds } \\
\# \text { vn } \\
\# \text { cck }\end{array}$ & $\begin{array}{c}4.51 \\
4036 \\
27.537 \mathrm{M}\end{array}$ & $\begin{array}{c}2.14 \\
2881 \\
18.816 \mathrm{M}\end{array}$ \\
\hline $\begin{array}{c}\text { rand-2-40-19-443-230- } \\
\text { fcd-1_ext } \\
\text { (sat) }\end{array}$ & $\begin{array}{c}\text { cpu seconds } \\
\text { \#vn } \\
\# \text { cck }\end{array}$ & $\begin{array}{c}27.285 \\
17707 \\
168.271 \mathrm{M}\end{array}$ & $\begin{array}{c}50.139 \\
49154 \\
451.828 \mathrm{M}\end{array}$ \\
\hline $\begin{array}{c}\text { rand-2-40-19-443-230- } \\
\text { fcd-11_ext } \\
\text { (sat) }\end{array}$ & $\begin{array}{c}\text { cpu seconds } \\
\text { \#vn } \\
\# \text { cck }\end{array}$ & $\begin{array}{c}313.233 \\
224053 \\
2008.373 \mathrm{M}\end{array}$ & $\begin{array}{c}41.839 \\
44490 \\
387.018 \mathrm{M}\end{array}$ \\
\hline
\end{tabular}

\subsection{Performance Evaluation of The Proposed Heuristics}

The proposed variable ordering uses MAC-AC3 and Revise methods to calculate the ratio of conflict to support counts. The Revise method is used to ensure a variable $X_{i}$ participated in a binary constraint is arc consistent with respect to the other variable $X_{j}$ participated in the same constraint. The Revise method works by simply testing every value in the domain of $X_{i}$ against every value in the domain of $X_{j}$, removing any value of $X_{i}$ that has no support in the domain of $X_{j}$. Therefore the complexity of Revise is bounded by the size of the domain of $X_{i}$ and $X_{j}$. Revise is usually implemented using a 2- 
level nested for loop. In the worst case scenario, every value in the domain of $X_{i}$ will have to be tested against every value in $X_{j}$. Therefore, the Revise method has $O\left(d^{2}\right)$ time complexity where $\mathrm{d}$ is the size of the largest domain.

The MAC-AC3 is an improvement over MAC-AC1 (Dechter, 2003). MAC-AC1 maintains arc consistency of the constraint network by calling Revise method for each pair of connected variables in the network. The Revise method is called until no value is deleted from any domain of variables. MAC-AC1 is not efficient because it wasted a lot of work on revising the arcs that are not affected by previous cycle of revise. All arcs are processed in every cycle regardless of whether the arc is affected by value deleted from the previous cycle. MAC-AC1 has $O\left(n e d^{3}\right)$ time complexity with $n$ variables, $e$ binary constraints and the maximum domain size of (Dechter, 2003). Every cycle of revising each binary constraint in the graph has the time complexity of $O\left(e d^{2}\right)$ since Revise method has $O\left(d^{2}\right)$ time complexity. According to the proof by (Dechter, 2003), there are a total of $n d$ possible cycles because in the worst case scenario each call to Revise may delete only one value from a domain and there are a total of $n d$ possible domain values. Therefore the time complexity of MAC-AC1 is $O\left(n e d^{3}\right)$.

MAC-AC3 improves the performance of MAC-AC1 by reprocessing only those arcs affected by the deleted values from the previous cycle. MAC-AC 3 achieves this by maintaining a queue of arcs to be processed. Initially, a queue is created to store all arcs in the network. Each arc is removed from the queue and processed by calling Revise method. This arc is put back to the queue only if the domain of its connected variables has changed as a result of calling Revise method to process the adjacent constraints. By doing this, each constraint can be processed by calling Revise method for at most $2 d$ 
times where $d$ is the size of the largest domain, because there is a maximum of $2 d$ values for any two connected variables that can be deleted (Dechter, 2003). For $e$ number of binary constraints, there will be $2 d e$ calls to the Revise method. Since each call to Revise method has $O\left(d^{2}\right)$ time complexity, taking only the fastest growing term, the time complexity of MAC-AC3 is therefore $O\left(e d^{3}\right)$. The maximum number of possible edges for a given graph with $n$ variables is $e=n(n-1) / 2$ or $(n 2-n) / 2$. Thus $O\left(e d^{3}\right)$ can also be written as $O\left(n^{2} d^{3}\right)$.

The proposed value ordering heuristic gathers information on conflict and support counts during forward checking stage. The standard forward checking heuristic has $O\left(e d^{2}\right)$ time complexity where $d$ is the largest domain size and $e$ is the number of constraints involved with future variables (Dechter, 2003). During forward checking, a value $v$ belongs to the current variable $X_{\text {cur }}$ is selected and instantiated. This value $v$ is tested against each of the $d$ values in the domains of each future connected variable for consistency. Each connected future variables is involving in $e_{i}$ constraints and therefore $e_{i} d$ tests is required for each connected future variables, and for all future connected variables, ed tests is required for each $v$, where $e$ is the sum of all $e_{i}{ }^{\prime} \mathrm{s}$ (Dechter, 2003). For a maximum domain size of $d$ values for $X_{c u r}, e d^{2}$ tests are required. Therefore, forward checking has $O\left(e d^{2}\right)$ or $O\left(n^{2} d^{2}\right)$ time complexity.

The implementation of the proposed heuristics uses the standard forward checking, revise and MAC-AC3 methods. The algorithms used to calculate the ratio of conflicts to supports count embedded in these methods all have constant time complexity of $O(1)$. Therefore, the proposed variable ordering heuristic using MAC-AC3 has $O\left(n^{2} d^{3}\right)$ time 
complexity and the proposed value ordering heuristic using forward checking has $O\left(n^{2} d^{2}\right)$ time complexity.

The space required to store the constraints network and the incompatible pair of values for each constraint is of $O\left(e d^{2}\right)$ where $e$, is the number of constraints in the problem and $d$, is the maximum domain size. The table of size $e$ is required for storing the arcs and for each arc a table of size $d^{2}$ is required to store the incompatible pairs. Therefore, it is of $O\left(e d^{2}\right)$ or $O\left(n^{2} d^{2}\right)$. The space required is not prohibitive and only one table is required. The space required to store the domain is of $O(n d)$ where $\mathrm{d}$ is the largest domain size and $n$ is the number of variables. Since the domain is required to be stored at every level of the tree for the current variable during backtracking search, therefore the complexity is of $O\left(n^{2} d\right)$ because there are $n$ levels of the tree. The space required to store the solution using one dimensional array is of $O(n)$ where $\mathrm{n}$ is the number of variables. The partial solution is also required to be stored at each level of the tree during backtracking search, therefore it is of $O\left(n^{2}\right)$ for the worst case. To keep track of the ratio of conflict to support counts a table space of $O\left(n^{2}\right)$ is also required where $\mathrm{n}$ is the number of variables.

For the proposed value ordering heuristic, the space complexity for storing the domain is $O\left(n^{2} d^{2}\right)$ and $O\left(n^{2} d\right)$ for storing the ratio of conflict to support counts. This is because of the look-ahead value ordering using forward-checking, where at each level of the search tree up to $d$ values are being explored. For each value explored, the domain and the ratio table is stored for future use. 
The time and space complexity for the proposed heuristics is of $O\left(e d^{2}\right)$ or $O\left(n^{2} d^{2}\right)$ for the worst case scenario. This complexity is not prohibitive for modern day computer memory.

\subsection{Conclusion}

In this chapter I have presented the experimental study conducted to evaluate the proposed heuristics. I explain the experimentation environment, evaluation criteria and CSP instances used to conduct this experimental study. A very clear picture has been given on how the environment is set up to conduct the experiment and how the performance is evaluated. An analysis of the experimental result is also given. It is clear from the experimental results that the proposed heuristic performs better than dom/ddeg when the problem gets larger and more difficult in general with very few exceptions. This is especially true from the experimental results obtained from testing frb series in table 45. The reason lies in the fact that as the search gets deeper many values are removed by MAC and the constraint tightness of the variable may change and differ significantly in terms of conflict and support counts. This is when the work of measuring the tightness by conflict to support counts gets pay off. For easy problems with many solutions the extra work is never paid off. The results from testing the Quasi-Random instaces Ehi series also show that the proposed heuristic is able to identify the hard part of the problem and reduce search time significantly as compared to dom/ddeg. Ehi instances are all unsatisfiable because they are embedded with a small unsatisfiable part. These instances are also very large. The proposed heuristic is able to prove that the problems have no solution with less than a second CPU time whereas dom/ddeg runs out of time limit. The dom/ddeg heuristic may not be precise in selecting the most constraint variable if the 
constraint tightness of relations are very different. The selection can get even less accurate if all variables have the same degree. By using the conflict to support counts, the proposed heuristic can precisely select the most constraint variable.

I have also tested the proposed value ordering heuristic. It is clear from the result that the proposed value ordering heuristic, when used together with the proposed variable ordering heuristic, can improve the performance for very large and difficult problems only. The results also show that the ranking strategy has saved the constraint checks and reduced the search space. However, the results also show that for easy or moderate problems with very few deadends and many acceptable values, the proposed value ordering heuristic is too costly. 


\section{Chapter 5}

\section{Conclusions and Future Work}

\subsection{Concluding Remarks}

In this thesis, I have proposed a weighted-degree heuristics using conflicts to supports ratio for dynamic variable ordering in CSPs. I have also proposed a dynamic value ordering heuristic using conflicts to supports ratio. The proposed value ordering heuristic can be used together with the proposed variable ordering heuristic. The experiments conducted on various benchmark problems from the authoritative website show that the proposed dynamic variable heuristic is comparable to the powerful generic dynamic variable ordering heuristic dom/ddeg. In some cases, the proposed dynamic variable ordering heuristic outperforms dom/ddeg with a wide margin. This is especially true in cases of Quasi-Random and randomly generated hard problems. However the proposed heuristic loses for easy and small problems. This shows that the extra effort for calculating the ratio of conflicts to supports is only worthwhile for large difficult problems. For moderately difficult problems, the proposed variable ordering heuristic sometimes performs very well, such as those cases in the patterned instances. However no heuristic can be perfect for all problems all the time. The experimental results also show that when the proposed value ordering heuristic is used together with the variable ordering heuristic, the performance improves for large random and difficult problems. For easy problem the value ordering is an extra performance cost not worth paying. 
One major drawback of the value ordering heuristic is that it is difficult to implement. The implementation requires one table for each value to cache the results obtained during the values ranking process for future use. The amount of space required for these tables can be very substantial for variables with large domain size. However, implementation using Java helps a lot because of the garbage collection feature in Java. Manipulating these tables during search also incurs some CPU overhead. For problems with many solutions this value ordering heuristic is a performance cost that is not worth paying. However, for large and difficult problems with fewer solutions the reward of ranking values could sometimes be very rewarding as shown in the experimental results.

\subsection{Future Work}

For future work, I would like to look into the possibility of optimize the value ordering heuristic. One possibility is to do value ordering only for upper levels of the tree. As the search gets to the lower levels of the tree most conflicting values would have been removed by MAC. Doing value ordering could be a waste of CPU time. Another possibility is to improve the implementation of the value ordering heuristic. I use arrays as data structures for storing domains, constraints and incompatible pairs of value in this implementation. However, other data structures may be more efficient for storing domain values. I would also like to look into the possibility of using intelligent backtracking instead of the standard backtracking for implementing the proposed heuristics.

In near future, many more instances from the website will also be tested with my proposed heuristics. I am also looking into the possibility of other methods of gathering information based on the structure of the problems. I also have in mind of using 
concurrent programming technique such as multithread programming to gather useful information before the main search starts.

\section{References}

Bacchus, F. (2000). Extending forward checking. In Proceedings of 6th International Conference on Principles and Practice of Constraint Programming, (pp. 35-51).

Bartak, R. (1998). Online guide to constraint programming. Retrieved December 5, 2014, from Roman Bartak's website: http://ktiml.mff.cuni.cz/ bartak/constraints/

Bartak, R. (2001). Theory and practice of constraint propagation. In Proceedings of the 3rd Workshop on Constraint Programming in Decision and Control, (pp. 7-14). .

Bayardo, R. J., \& Schrag, R. C. (1997). Using csp look-back techniques to solve realworld sat instances. In Proceedings of the Fourteenth National Conference on Artificial Intelligence and Ninth Conference on Innovative Applications of Artificial Intelligence AAAI'97, (pp. 203-208).

Bessiere, C., \& Regin, J. C. (1996). MAC and combined heuristics: Two reasons to forsake FC (and cbj?) on hard problems. In Proceedings of the Second International Conference on Principles and Practice of Constraint Programming, (pp. 61-75).

Boussemart, F., Hemery, F., \& Lecoutre, C. (2005). Description and representation of the problems selected for the first international constraint satisfaction solver competition. In 2nd International Workshop on Constraint Propagation And Implementation held with CP'05(CPAI'05), (pp. 7-26).

Boussemart, F., Hemery, F., Lecoutre, C., \& Sais, L. (2004). Boosting systematic search by weighting constraints. In Proceedings of the 16th European Conference on Artificial Intelligence, ECAI'2004, (pp. 146-150).

Brelaz, D. (1979). New methods to color vertices of a graph. Communications of the ACM, 22(4), 251-256.

Dechter, R. (1990). Enhancement schemes for constraint processing: Backjumping, learning, and cutset decomposition. Artificial Intelligence, 41(3), 273-312.

Dechter, R. (2003). Constraint Processing. San Francisco: Morgan Kaufmann. 
Freuder, E. C., \& Quinn, M. J. (1985). Taking advantage of stable sets of variables in constraint satisfaction problems. In Proceedings of the 9th International Joint Conference on Artificial Intelligence, (pp. 1076-1078).

Frost, D. H. (1997). Algorithms and heuristics for constraint satisfaction problems. $\mathrm{PhD}$ thesis, University of California, Irvine.

Frost, D. H., \& Dechter, R. (1994). In search of the best constraint satisfaction search. In Proceedings of the 12th National Conference, (pp. 301-306).

Frost, D. H., \& Dechter, R. (1995). Look-ahead value ordering for constraint satisfaction problems. In Proceedings of the Fourteenth International Joint Conference on Artificial Intelligence, IJCAI 95, (pp. 572-578).

Gaschnig, J. (1978). Experimental case studies of backtrack vs. Waltz-type vs. new algorithms for satisfying assignment problems. In Proceedings of the Second Canadian Conference on Artificial Intelligence, (pp. 268-277).

Geelen, P. A. (1992). Dual viewpoint heuristics for binary constraint satisfaction problems. In Proceedings of the 10th European Conference on Artificial Intelligence, ECAI'92, (pp. 31-35).

Gomes, C., \& Walsh, T. (2006). Randomness and structure. In Handbook of Constraint Programming (pp. 639-664). Elsevier.

Grimes, D., \& Wallace, R. J. (2007). Learning to identify global bottlenecks in constraint satisfaction search. In Proceedings of the Twentieth International Florida Artificial Intelligence Research Society Conference, (pp. 592-597).

Grimes, D., \& Wallace, R. J. (2007). Sampling strategies and variable selection in weighted degree heuristics. In Principles and Practice of Constraint Programming-CP 2007 (pp. 831-838). Springer Berlin Heidelberg.

Haralick, R. M., \& Elliott, G. L. (1980). Increasing tree search efficiency for constraint satisfaction problems. Artificial intelligence, 14(3), 263-313.

Jafari, B. (2010). Using Non-Systematic Algorithms for Variable and Value Ordering in CSPS. Master Thesis, 2010, University of Regina.

Kumar, V. (1992). Algorithms for constraint-satisfaction problems: A survey. AI Magazine, 13(1), 32-44.

Mehta, D., \& van Dongen, M. (2005). Static value ordering heuristics for constraint satisfaction problems. In Proceedings of the Second International Workshop on Constraint Propagation And Implementation, (pp. 49-62). 
Mouhoub, M., \& Jafari, B. (2011). Heuristic techniques for variable and value ordering in csps. In Proceedings of the 13th Annual Conference on Genetic and Evolutionary Computation, (pp. 457-464).

Muller, H. (2005). Static and dynamic variable sorting strategies for backtracking-based search algorithms. 19th Workshop on Constraint Logic Programming, (pp. 99-110).

Nadel, B. (1989). Precision complexity analysis: A framework for deriving exact-case results. Technical Report CSC-89-004, Wayne State University.

Prosser, P. (1993). Hybrid algorithms for the constraint satisfaction problem. Computational intelligence, 9(3), 268-299.

Smith, B. (1995). A tutorial on constraint programming. Technical Report, University of Leeds.

Smith, B. M., \& Grant, S. A. (1997). Trying harder to fail firrst. Technical Report, University of Leeds.

Stallman, R. M., \& Sussman, G. J. (1977). Forward reasoning and dependency-directed backtracking in a system for computeraided circuit analysis. Artificial intelligence, 9(2), 135-196.

Xu, K. (2015). Forced satisfiable csp and sat benchmarks of model rb - hiding solutions with growing domains. Retrieved June 25, 2015, from http://www. nlsde.buaa.edu.cn/ kexu/benchmarks/benchmarks.htm 